Provided for non-commercial research and education use. Not for reproduction, distribution or commercial use.

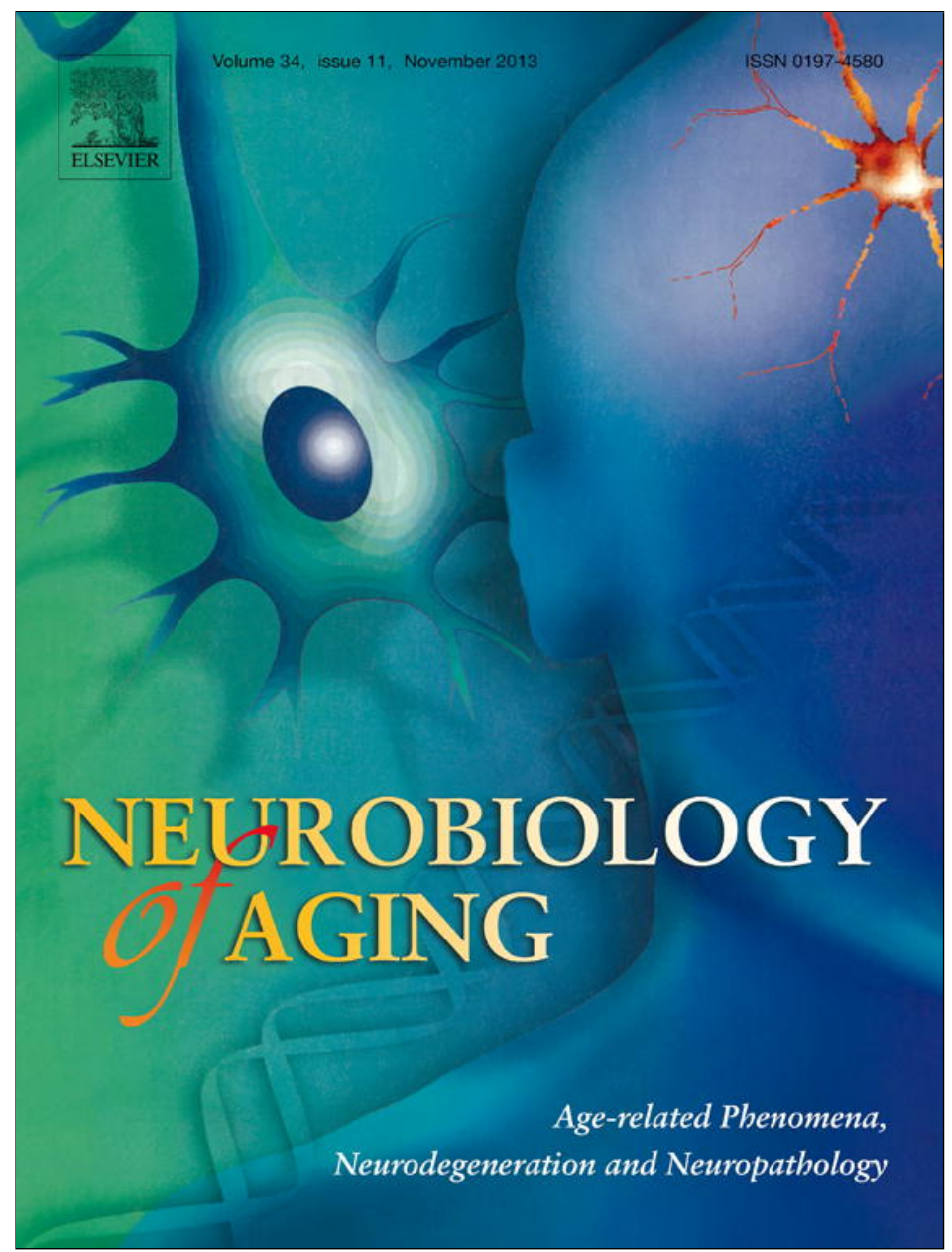

This article appeared in a journal published by Elsevier. The attached copy is furnished to the author for internal non-commercial research and education use, including for instruction at the authors institution and sharing with colleagues.

Other uses, including reproduction and distribution, or selling or licensing copies, or posting to personal, institutional or third party websites are prohibited.

In most cases authors are permitted to post their version of the article (e.g. in Word or Tex form) to their personal website or institutional repository. Authors requiring further information regarding Elsevier's archiving and manuscript policies are encouraged to visit:

http://www.elsevier.com/authorsrights 


\title{
Impact of N-tau on adult hippocampal neurogenesis, anxiety, and memory
}

\author{
Andrea Pristerà ${ }^{\mathrm{a}}$, Daniele Saraulli ${ }^{\mathrm{b}}$, Stefano Farioli-Vecchioli ${ }^{\mathrm{a}}$, Georgios Strimpakos ${ }^{\mathrm{a}}$, Marco Costanzi ${ }^{\mathrm{a}, \mathrm{c}}$, \\ Maria Grazia di Certo ${ }^{a}$, Sara Cannas ${ }^{a}$, Maria Teresa Ciotti ${ }^{a}$, Felice Tirone ${ }^{a}$, Elisabetta Mattei ${ }^{a}$, \\ Vincenzo Cestari $^{\mathrm{a}, \mathrm{d}}$, Nadia Canu ${ }^{\mathrm{e}, \mathrm{f}, *}$ \\ ${ }^{a}$ Institute of Cellular Biology and Neurobiology, National Research Council and Fondazione Santa Lucia, Rome, Italy \\ ${ }^{\mathrm{b}}$ Department of Psychology, Sapienza University of Rome, Rome, Italy \\ ${ }^{c}$ Department of Human Sciences Libera Universitá Maria Santissima Assunta (LUMSA), Rome, Italy \\ ${ }^{\mathrm{d}}$ Department of Psychology and "Daniel Bovet" Center, Sapienza University of Rome, Rome, Italy \\ e Department of System Medicine, University of Rome "Tor Vergata", Rome, Italy \\ ${ }^{\mathrm{f}}$ Institute of Cellular Biology and Neurobiology, National Research Council, Rome, Italy
}

\section{A R T I C L E I N F O}

\section{Article history:}

Received 7 October 2012

Received in revised form 11 April 2013

Accepted 8 May 2013

Available online 12 June 2013

\section{Keywords:}

tau

Adult hippocampal neurogenesis

Neuronal progenitor cell

Dentate gyrus

Episodic-like memory

Anxiety

\begin{abstract}
A B S T R A C T
Different pathological tau species are involved in memory loss in Alzheimer's disease, the most common cause of dementia among older people. However, little is known about how tau pathology directly affects adult hippocampal neurogenesis, a unique form of structural plasticity implicated in hippocampusdependent spatial learning and mood-related behavior. To this aim, we generated a transgenic mouse model conditionally expressing a pathological tau fragment (26-230 aa of the longest human tau isoform, or N-tau) in nestin-positive stem/progenitor cells. We found that $\mathrm{N}$-tau reduced the proliferation of progenitor cells in the adult dentate gyrus, reduced cell survival and increased cell death by a caspase3 -independent mechanism, and recruited microglia. Although the number of terminally differentiated neurons was reduced, these showed an increased dendritic arborization and spine density. This resulted in an increase of anxiety-related behavior and an impairment of episodic-like memory, whereas less complex forms of spatial learning remained unaltered. Understanding how pathological tau species directly affect neurogenesis is important for developing potential therapeutic strategies to direct neurogenic instructive cues for hippocampal function repair.
\end{abstract}

(C) 2013 Elsevier Inc. All rights reserved.

\section{Introduction}

The neurobiological substrate of learning and memory resides in modifications of synaptic strength and structural changes of neural networks activated during learning. Adult neurogenesis, the birth and development of new neurons in the adult brain, represents a unique form of structural and functional plasticity found in the hippocampal dentate gyrus (DG) and subventricular zone (SVZ) of lateral ventricles. DG is a critical structure for learning and memory functions (Deng et al., 2010; Emsley et al., 2005; Inokuchi et al., 2011). The capacity to encode and retain memories is severely compromised in dementia. Alzheimer's disease (AD) is the most common cause of dementia in elderly people, characterized by synaptic pathology, intracellular accumulation of tau protein in

\footnotetext{
A.P., D.S., and S.F.-V. contributed equally to this work.

* Corresponding author at: Department of Systems Medicine, University of Rome 'Tor Vergata', Via Montpellier 1, 00133 Rome, Italy; and Institute of Cellular Biology and Neurobiology, National Research Council, Italy, Via del Fosso di Fiorano, 64, 00143 Rome, Italy. Tel.: 003906 501703233; fax: 003906501703313.

E-mail addresses: n.canu@inmm.cnr.it, nadiacanu@tiscali.it (N. Canu).
}

neurofibrillary tangles, tau spreading, extracellular accumulation of $\beta$-amyloid in senile plaques, and loss of specific neuronal populations. Although these lesions and cognitive dysfunction are poorly correlated, the former may be directly involved, individually or in combination, in damaging brain plasticity in vulnerable regions of the brain (Ittner et al., 2010).

Abnormalities in tau protein-such as hyperphosphorylation, mutation, truncation, oligomerization, aggregation, as well as imbalance in isoform ratios (Chatterjee et al., 2009; Jackson et al., 2002; Santacruz et al., 2005) - are sufficient to cause synaptic dysfunction, neurodegeneration, and dementia. Among tau abnormalities, we focused on the N-terminal 26-230 tau fragment $(\mathrm{N}-$ tau). Different lines of evidence support a role for this fragment in the impairment of neuronal plasticity and neurodegeneration: (1) $\mathrm{N}$-tau is generated by caspase and/or calpain in different in vitro and in vivo models of AD (Canu et al., 1998; Corsetti et al., 2008; Park and Ferreira, 2005); (2) it is expressed in human AD brains (Amadoro et al., 2012; Rohn et al., 2002); (3) it is toxic when overexpressed in primary neuronal cultures (notably, the mechanism of toxicity involves the NMDAR and the signal transduction 
pathways linked to the activation of this receptor, like that of mitogen-activated protein [MAP] kinases) (Amadoro et al., 2006); and (4) a similar tau product, originating by an alternative splicing of exon 6, abundant in fetal brains and in CA1/CA3 pyramidal cells and DG granular cells of adult hippocampus, is supposed to be involved in the morphogenesis of synapses and neuronal networks, as well as in morphogenetic apoptosis (Luo et al., 2004).

The subgranular zone (SGZ) of the DG is characterized by the presence of progenitor cells that produce, by a highly regulated process, glutamatergic neurons for the entire life of the organism. These neurons populate the granule cell layer of the DG and become functionally integrated into the existing DG circuitry (Kempermann et al., 2004a, 2004b). Studies have correlated adult DG neurogenesis with cognitive function and hippocampus-mediated learning and memory (Deng et al., 2010). Moreover, it is believed that the abnormal regulation of adult hippocampal neurogenesis might account for cognitive deterioration in neurodegenerative diseases associated with dementia (Lazarov et al., 2010; Mu and Gage, 2011).

Alterations in the amyloid precursor protein (APP) and tau metabolism may indirectly perturb the permissive cues within the neurogenic niche that drive the production of new neurons and their subsequent integration into the neurocircuitry of the brain (Ghosal et al., 2010; Haughey et al., 2002; Rodriguez et al., 2008). Less studied, however, are the direct effects on adult hippocampal neurogenesis of proteins that, as tau, regulate the morphogenesis and functional integration of new neurons (Bullmann et al., 2007; Fuster-Matanzo et al., 2012; Hong et al., 2010).

With this aim, we investigated the link between pathological $\mathrm{N}$ tau and adult neurogenesis, by means of a novel conditional transgenic mouse model expressing $\mathrm{N}$-tau in neuronal precursor cells. Here we report the effect of $\mathrm{N}$-tau expression in neuronal precursor cells, and we demonstrate that significant loss of nestinpositive neuronal stem cells and terminally differentiated newborn neurons was coupled with increased anxiety-related behavior in stressful conditions and deficit in episodic-like memory, both documented at the early onset of Alzheimer's disease.

\section{Methods}

\subsection{Mouse lines and genotyping}

The bi-transgenic nestin-rtTA/TRE-N-tau mouse line (henceforth referred to as TgN-tau) is the progeny of 2 mouse lines, each carrying a transgene: (1) nestin-rtTA transgene, encoding the tetracycline-regulated transactivator (rtTA) protein driven by the rat nestin intron II enhancer/promoter, previously generated (Mitsuhashi et al., 2001), which restricts reporter gene expression to neuronal tissue (Fukuda et al., 2003, Kronenberg et al., 2003; Yamaguchi et al., 2000); and (2) TRE-N-tau transgene, carrying the 26-230 fragment of the longest human tau isoform under the control of the tetracycline-responsive elements (TRE) (Gossen et al., 1995).

The N-tau fragment was cloned into pTRE-shuttle2 vector (Clontech, Mountain View, CA, USA) to obtain pTRE-N-tau vector. To generate TRE-N-tau mice, the 1.3-Kb fragment-containing 7 copies of TRE promoter, followed by the minimal cytomegalovirus promoter, the $\mathrm{N}$-tau sequence and the SV40 poly(A)-was excised from the pTRE-N-tau vector by Nrul/BseRI digestion and used for microinjecting zygotes derived from hormonally primed 6- to 8week-old B6D2F1 (C57BL/6 x DBA/2) female mice (Charles River) mated with fertile males of the same strain.

The TgN-tau mice were isogenic, having been previously interbred for 6 or more generations. The genotypization was performed by polymerase chain reaction analysis on genomic DNA extracted from tail biopsy using different sets of primers, to analyze rtTA and
$\mathrm{N}$-tau transgene respectively. Primers used to identify nestin-rtTA transgenic mice (amplified $991 \mathrm{bp}$ of the tTA transgene) were as follows: forward $5^{\prime}$-AAGTAAAGTGATTAACAGCGC-3' and reverse (5'-CTACCCACCGTACTCGTC-3'), whereas primers used to identify TRE-N-tau mice were: forward $5^{\prime}$ - GGATCCTCTAGTCAGCTGACG $-3^{\prime}$ (nucleotide 720-744 of pTRE shuttle vector 2) and reverse $5^{\prime}$ ACGGACGCACTGCCACCTTCTT-3' (nucleotide 672-690 of human tau 1-441mRNA).

Animals were housed in standard breeding cages under a 12 hour light-dark schedule at a constant temperature of $21^{\circ} \mathrm{C}$, and underwent behavioral testing between 2 and 5 PM in sound-insulated rooms. All procedures involving mice were conducted in accordance with the Istituto Superiore di Sanità (Italian Ministry of Health) and current European Ethical Committee (Directive 86/609/ ECC) guidelines. All efforts were made to minimize the numbers of animal used and their suffering.

\subsection{Animal treatment}

The N-tau transgene was activated in TgN-tau mice (TgN-tau ON mice) at P60 by doxycycline (Dox; $2 \mathrm{mg} / \mathrm{mL}$; Sigma Aldrich, St. Louis, MO, USA) supplied in drinking water containing 2.5\% sucrose. Dox-free animals (TgN-tau OFF mice [bi-transgenic mice in which the TRE-N-tau transgene was not activated], and WT mice [the progeny of crosses between nestin-rtTA and TRE-N-tau transgenic mice in which none of transgenes were inherited]) were given only $2.5 \%$ sucrose in drinking water and used as controls. Four additional control groups (2 groups of Nestin-rtTA mice, Dox-free and Doxtreated, respectively; and 2 groups of TRE-N-tau mice, Dox-free and Dox-treated respectively) were used. At P90, mice received 5 daily bromodeoxyuridine (BrdU; $95 \mathrm{mg} / \mathrm{kg}$ i.p.; Sigma Aldrich, St. Louis, MO, USA) injections to study neurogenesis. Immunohistochemical analyses were performed after BrdU treatment, at P95 and P116.

\subsection{Immunohistochemistry, BrdU labeling, immunofluorescence, and TUNEL analysis}

Mice were anesthetized with $400 \mathrm{mg} / \mathrm{kg}$ choral hydrate (Sigma Aldrich, St. Louis, MO, USA) and transcardially perfused with a phosphate-buffered solution of $4 \%$ paraformaldehyde. Their brains were removed, left in the fixative overnight, equilibrated in sucrose $30 \%$, and cryopreserved at $-80{ }^{\circ} \mathrm{C}$.

Brains embedded in Tissue-Tek OCT (Sakura Finetek Europe B.V) were cut (coronal section, $20-\mu \mathrm{m}$ thickness) on cryomicrotome (Leica CM $1850 \mathrm{UV}$ ) at $-25^{\circ} \mathrm{C}$. Sections were then processed immunohistochemically for multiple labeling with BrdU and other cellular markers using fluorescent methods. BrdU incorporation was visualized by denaturing DNA through pretreatment of sections with $2 \mathrm{~N} \mathrm{HCl}$ for $45 \mathrm{~min}$ at $37^{\circ} \mathrm{C}$, followed by $0.1 \mathrm{~mol} / \mathrm{L}$ sodium borate buffer, $\mathrm{pH} 8.5$, for 10 minutes.

Primary antibodies were as follows: rat monoclonal antibody against BrdU (MCA2060; 1:400; AbD Serotech; Raleigh, NC, USA); mouse monoclonal antibody against nestin (USAMAB353; 1:100; Chemicon International, Temecula, CA, USA), NeuN (MAB377; 1:300; Chemicon International), tau (MN-100, clone HT7, 1:300; Thermo-Fisher Pierce, Rockford, IL, USA); chicken polyclonal antibody against nestin (PA1-46338 1:1000; Thermo-Fisher Pierce); goat polyclonal antibody against Glial Fibrillary Acidic Protein (GFAP; Sc-6170, 1:300; Santa Cruz Biotechnology, Santa Cruz, CA), Dcx (Sc-8066, 1:300; Santa Cruz Biotechnology), Iba-1 (ab5076, 1:100; Abcam, Cambridge, UK); rabbit polyclonal antibody against Dcx (ab18723; 1:200; Abcam); rabbit monoclonal anti-Ki67 (SP6; 1:200; LabVision Corporation), rabbit polyclonal antibody against anti-cleaved caspase-3 (9961, 1:100; Cell Signaling Technologies, Danvers, MA, USA). After overnight incubation, primary antibody 
staining was revealed using fluorescence-conjugated secondary donkey antibodies (Jackson ImmunoResearch Europe Ltd).

Terminal deoxynucleotidyl transferase-mediated biotinylated UTP nick end labeling (TUNEL) was performed according to the manufacturer's instructions (ApopTag Red in situ apoptosis detection kit; Millipore) on cryostat sections.

Images of the immunostained sections were obtained by laser scanning confocal microscopy using a TCS SP5 microscope (Leica Microsystem). All analyses were performed in sequential scanning mode to rule out cross-bleeding between channels.

\subsection{Quantification of cell number and volumes}

Quantitative analysis of cell populations was performed by means of design-based (assumption-free, unbiased) stereology. Slices were collected using systematic random sampling. The brain was sliced coronally in rostro-caudal direction, thus including the entire DG. Approximately 200 coronal sections of $20 \mu \mathrm{m}$ were obtained from each brain; about 1 -in-10 series of sections (each slice thus spaced $200 \mu \mathrm{m}$ apart from the next) were analyzed by confocal microscopy and used to count, by unbiased stereological method, the number of cells expressing the indicated markers throughout the whole rostro-caudal extent of the DG. The total estimated number of cells within the DG, positive for each of the indicated markers or combination of markers, was obtained by multiplying the average number of positive cells per section by the total number of $20 \mu \mathrm{m}$ sections comprising the entire DG (spaced $200 \mu \mathrm{m}$ ) (Farioli-Vecchioli et al., 2008; Gould et al., 1999; Jessberger et al., 2005; Kee et al., 2007). Double and triple labeling was confirmed by z-series of entire nucleus or cell in question. An investigator blinded to the different experimental design settings performed cell counting and proportional analyses. At least 3 animals per group were analyzed.

Stereological analysis of volumes was performed, analyzing every sixth section in a series of $40-\mu \mathrm{m}$ coronal sections (spaced $240 \mu \mathrm{m}$ ). Volume was determined by summing the traced areas of the DG or the hippocampus and multiplying this result by the distance between the sections analyzed $(240 \mu \mathrm{m})$.

Measurements of positive cells and areas were obtained by computer-assisted analysis using the I.A.S. software (Delta Sistemi, Rome, Italy).

\subsection{Assay of $\beta$-galactosidase activity}

$\beta$-Galactosidase activity of the $\beta$-geo reporter gene fused to the nestin-rtTA transgene was performed as previously described (Farioli-Vecchioli et al., 2008).

\subsection{Retrovirus-GFP labeling}

The murine Moloney leukemia virus-based vector CAG-green fluroescence protein (GFP) (Zhao et al., 2006) was used to infect only dividing cells at the moment of in vivo delivery. Retroviruses were propagated by transiently cotransfecting CAG-GFP with pHCMV-G vector, which expresses the VSV-G protein (FarioliVecchioli et al., 2008; Gaiano et al., 1999) in the packaging cell line Phoenix (the latter being a human embryonic kidney cell line that stably expresses the gag and pol proteins of Moloney murine leukemia virus). Cells at approximately $90 \%$ confluence in $90-\mathrm{mm}$ dishes were transfected with $11.5 \mu \mathrm{g}$ of CAG-GFP and with $13.5 \mu \mathrm{g}$ of pHCMV-G, using calcium phosphate precipitation. Virus-containing supernatant was harvested 36,48 , and 60 hours after the start of transfection. Frozen stocks were pooled and the virus was concentrated by centrifugation for 1.5 hours at $60,000 \times g$. The concentrated $\left(10^{8-9} \mathrm{cfu} / \mathrm{mL}\right)$ was bilaterally infused $(1.5 \mu \mathrm{L}$ at 0.32
$\mathrm{mL} / \mathrm{min}$ ) into the DG of anesthetized mice at P95 (coordinates: $2 \mathrm{~mm}$ posterior and $1.5 \mathrm{~mm}$ lateral from bregma and $2.3 \mathrm{~mm}$ ventral from skull).

\subsection{Dendritic growth and spine density of GFP-positive neurons}

Dendritic analysis of GFP-positive neurons was performed by acquiring z-series of 15 to 25 optical sections at 1 - to $1.5-\mu \mathrm{m}$ intervals with a $\times 40$ oil lens, with the confocal system TCS-SP5 (Leica Microsystem). Two-dimensional projections at maximum intensity of each z-series were generated with the LAS AF software platform (Leica Microsystem) in the TIFF format, and files were imported in the I.A.S. software (Delta Systems) to measure dendritic length. The number of branching points was counted manually in the same images. For each data point, 20 to 30 cells from 3 mice per group were analyzed. From the same GFP-positive neurons, the spines on dendritic processes were imaged by acquiring z-series of 25 to 35 optical sections at $0.5-\mu \mathrm{m}$ intervals with $\mathrm{a} \times 63$ apochromatic oil lens, and a digital zoom of 3 . The number of spines was counted manually on 2-dimensional projections obtained by the LAS AF software. The linear spine density was then calculated by dividing the total number of spines by the length of the corresponding dendritic process.

\subsection{Behavioral tests}

TgN-tau ON ( $\mathrm{n}=36)$, TgN-Tau OFF ( $\mathrm{n}=44)$, WT $(\mathrm{n}=21)$, and Nestin-rtTA dox-treated $(\mathrm{n}=25)$, male mice aged between 3 and 5 months were used for behavioral evaluation.

\subsubsection{Elevated plus maze}

The elevated plus maze test was performed in an elevated maze (60 $\mathrm{cm}$ from the ground), made of gray Plexiglas, with 4 arms (5 $\times$ $30 \mathrm{~cm}$ ) extending from a central platform. Two opposite arms were enclosed by walls ( $15 \mathrm{~cm}$ high), whereas 2 were open. Two different experimental settings were prepared, for subsequent testing of independent groups of mice. A dimly lit ( $\sim 50$ lux), silent room housed the apparatus for the non-threatening condition, which was successively turned into a brightly lit one ( $\sim 500$ lux $)$, with direct lighting of the apparatus and moderate background noise, for the fearful condition. The mice were placed in the center of the apparatus and allowed to explore it for 5 minutes, to assess the relative occupancy of the open versus enclosed arms. The behavior of mice was analyzed by EthoVision software (Noldus Information Technology, Wageningen, the Netherlands).

\subsubsection{Light/dark emergence test}

The light/dark emergence test was conducted as previously described (Revest et al., 2009), with minor modifications. A darkgray plastic cylinder ( $15 \mathrm{~cm}$ deep, $7 \mathrm{~cm}$ in diameter) was placed lengthwise along a wall of a squared arena $(80 \times 80 \times 30 \mathrm{~cm})$. The mice were placed into the cylinder and tested for 10 minutes under bright light conditions ( $\sim 800$ lux), to assess the time spent inside the cylinder and the distance traveled in the lighted zone of the apparatus. The behavior of the mice was analyzed by EthoVision software (Noldus Information Technology, Wageningen, the Netherlands).

\subsubsection{Water maze (reference)}

Both the reference version and the delayed matching-to-place version of the Morris Water Maze were performed in a circular swimming pool ( $130 \mathrm{~cm}$ in diameter), filled with opaque water that was maintained at a constant temperature of $25^{\circ} \mathrm{C} \pm 1{ }^{\circ} \mathrm{C}$, located in a room containing prominent extra-maze cues. For the reference version, the mice were pre-trained to a visible platform for 2 days, to exclude motor deficits or weakening in visual acuity. The training 
consisted of 24 trials ( 4 trials per day, lasting a maximum of 60 seconds, with an intertrial interval of approximately 30 minutes), with the platform (12 cm in diameter) left in the same position in the pool. A probe test (60 seconds) was also performed, 24 hours after the completion of the last training session, by removing the platform from the pool and leaving the mice swim for 60 seconds. The behavior of the mice was analyzed by EthoVision software (Noldus Information Technology, Wageningen, the Netherlands).

\subsubsection{Water maze (delayed matching-to-place)}

The delayed-matching-to-place task was conducted as previously described (Chen et al., 2000; Zeng et al., 2001), with minor modifications. A first training session started in which the mice were trained to navigate to the hidden platform until reaching a rigorous performance criterion of 3 trials in a row with an average escape latency of less than 20 seconds, or until completing a maximum of 24 trials ( 8 trials per day, lasting a maximum of 90 seconds, with an intertrial interval of approximately 10 minutes). The day after the completion of the first training session, a new session started in which mice were trained to a different platform location, with the same procedure. This protocol was repeated until a total of 5 platform locations were learned. Animals that reached the criterion in less than 5 trials were still trained until completing a series of 5 trials, to collect escape latency data of all mice for the first 5 trials. The behavior of the mice was analyzed by EthoVision software (Noldus Information Technology).

Additional behavioral methods are available as supplementary material.

\section{Results}

\subsection{Doxycycline treatment in bi-transgenic $N$-tau mice induces expression of $\mathrm{N}$-tau in nestin-positive neuronal precursor cells}

$\mathrm{N}$-tau conditional expression was induced in nestin-expressing adult hippocampal stem and progenitor cells (Kempermann et al., 2004a, 2004b) of TgN-tau mice from P60 through administration of Dox (Fig. 1A). The analysis of the expression of transgenic N-tau

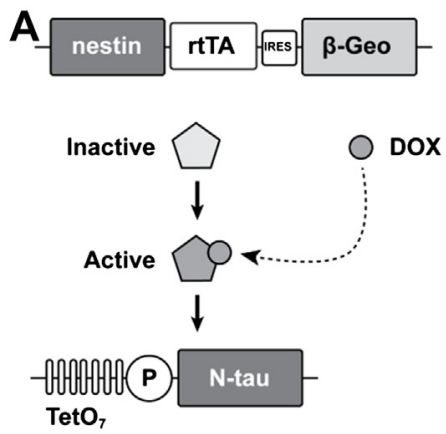

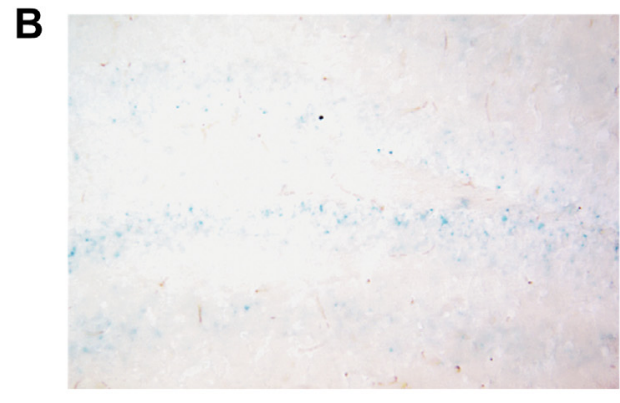

TgN-tau OFF

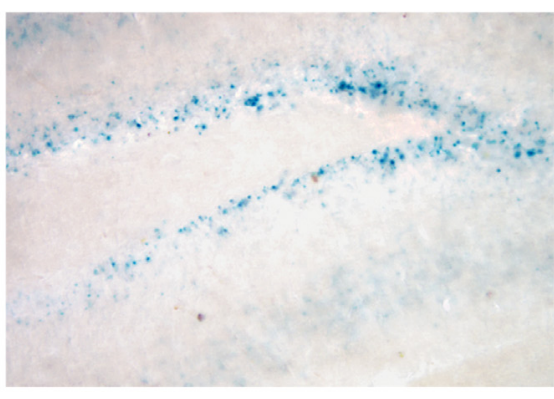

TgN-tau ON
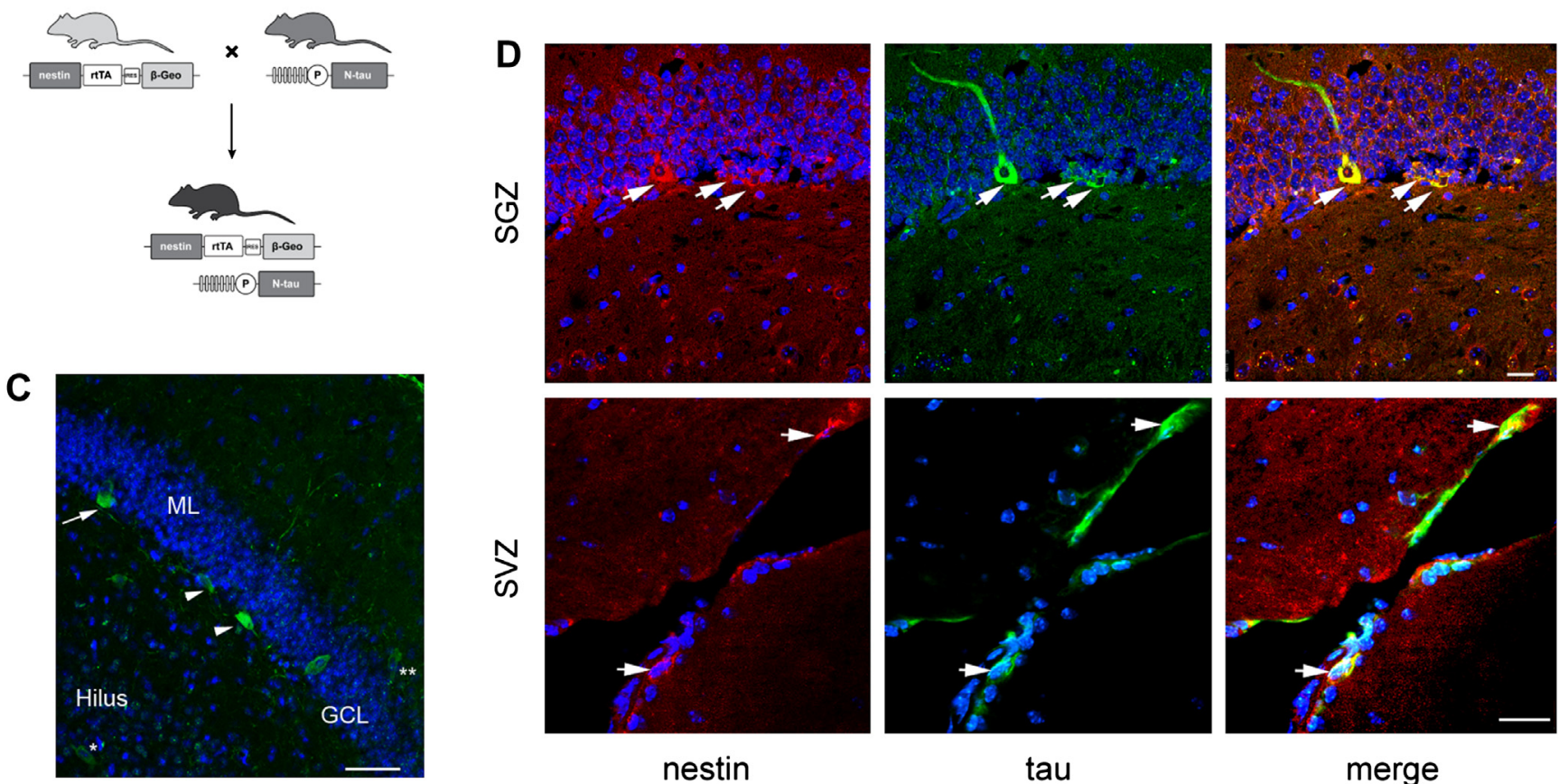

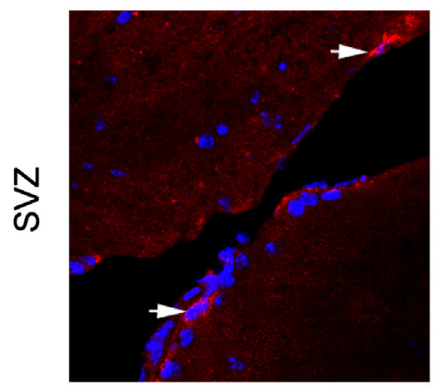

nestin

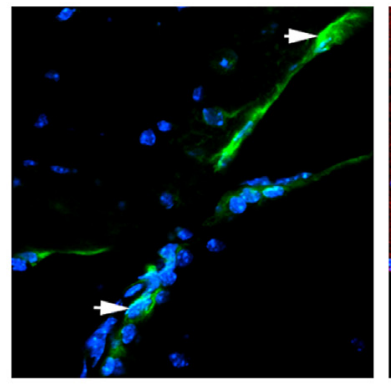

tau

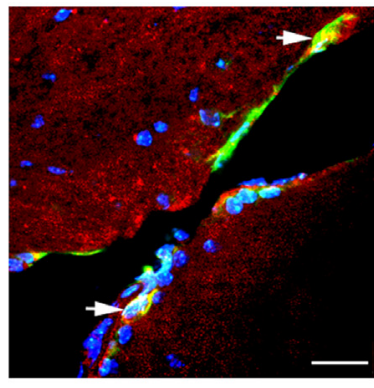

merge

Fig. 1. Inducible expression of N-tau protein in neuronal precursor using Tet-On system. (A) Schematic representation of Tet-On-inducible gene expression system. The rtTA protein is under the control of the rat nestin II enhancer promoter. Doxycycline (Dox) activates the rtTA protein, which binds to 7 TetON sequence (TRE) to drive the transcription of N-tau transgene. Approach to obtain Dox-induced expression of $\mathrm{N}$-tau in neuronal precursor in vivo using double transgenic nestin-rtTA/TRE-N-tau mice. (B) Selective targeting to the dentate gyrus (DG) in P95 mice of N-tau transgene (X-gal blue staining, activated since P60 by Dox administration). (C) Confocal illustration of N-tau expression in DG of TgN-tau ON mice. Immunofluorescence with tau monoclonal antibody (HT7) specific for human tau (green) reveals N-tau expression in subgranular zone (SGZ). Both putative type 1 cell with dendritic-like process extending to the outer granular cell layer (GCL) and non-process-bearing putative type 2 cells (arrowhead) are positive for N-tau. Note the presence of positive $\mathrm{N}$-tau cells in the hilus $\left({ }^{*}\right)$ and at the border of the molecular layer (ML) $\left(^{* *}\right)$. Scale bar, $50 \mu \mathrm{m}$. (D) Confocal illustration showing that nestin-positive neuronal precursors (red, arrow) are positive for N-tau (green) in both SGZ and SVZ. Scale bar, $20 \mu \mathrm{m}$. Hoechst nuclear counterstaining is shown in blue. 
in $\mathrm{P95}$ mice with active N-tau transgene (TgN-tau ON mice) indicated targeting to the DG, as visualized by X-gal staining, which revealed the $\beta$-galactosidase activity of the $\beta$-geo reporter gene fused to the nestin-rtTA transgene (Fig. 1B). In TgN-tau ON mice, the immunostaining with human specific tau antibody (HT7) revealed the presence of $\mathrm{N}$-tau in nestin-positive progenitor cells, namely, putative type 1 cells, with long processes extending to the outer granule cell layer and molecular layer, and putative type 2 cells, with a large round or ovoid soma and short cytoplasmic extension oriented tangentially (Fig. 1C and D). Moreover, N-tau-positive cells were detected in the middle granule cell layer, at the border of the molecular layer, and in the hilus, where nestin-positive cells also reside (Filippov et al., 2003) (Fig. 1C), and in SVZ of the lateral ventricles (Lugert et al., 2010) (Fig. 1D).

\subsection{N-tau expression decreases hippocampal neurogenesis}

To follow the progeny of proliferating cells, 30 days after the activation of the $\mathrm{N}$-tau transgene, mice received 5 daily injections (P90-P94) of permanent S-phase marker BrdU. Immunohistochemical analyses performed at P95 showed a significant decrease ( $\sim 25 \% ; p=0.016$ ) of the number of BrdU-labeled cells in the DG of TgN-tau ON mice compared to TgN-tau OFF (Fig. 2A and B). Similarly, the number of cells in SGZ positive for Ki67 (a protein associated with cell proliferation) was significantly reduced in TgN-tau ON mice compared to TgN-tau OFF ( 30\%; $p<0.005)$ (Fig. $2 \mathrm{C}$ ). These data indicate that cell proliferation is reduced by one-third in the SGZ of TgN-tau ON mice. A similar trend was observed in the SVZ of TgN-tau ON mice (Fig. S1) consistent with the Dox-induced $\mathrm{N}$-tau expression in this neurogenic niche.

Furthermore, no significant difference in the number of BrdUpositive cells was observed between different control groups (nestin-rtTA, TRE-N-tau, and WT), treated either with vehicle or Dox, and TgN-tau OFF (Fig. S2). For the sake of maximum clarity, only comparison between TgN-tau OFF and TgN-tau ON mice are shown. Data regarding all other control groups are reported in Supplementary Data.

\section{3. $N$-tau expression influences the lineage progression of the newly generated cells}

To determine whether the reduced cell proliferation observed in TgN-tau ON mice resulted from a significant decrease in the number of specific precursor cells or, alternatively, by a overall reduced proliferative activity, we quantified the absolute number of all newborn cell populations.

The glial fibrillary acidic protein (GFAP)-expressing astroglia rarely divide neural stem cells, undergoing asymmetric division to give rise to 1 daughter stem cell and 1 daughter neuronal lineage restricted progenitor cell (Seri et al., 2001). These stem cells, defined as type 1 (Kempermann et al., 2004a, 2004b), are identified by the expression of nestin, GAFP, Sox2, and BLBP (Filippov et al., 2003; Fukuda et al., 2003; Graham et al., 2003; Lugert et al., 2010). We found that, in TgN-tau ON mice, the number of $\mathrm{BrdU}^{+}$, $\mathrm{GFAP}^{+} /$nestin ${ }^{+}$cells, corresponding to 1 - to 5-day-old type 1 cells, decreased significantly ( $\sim 50 \% ; p=0.002$ ) (Fig. 2D).

Next, we analyzed the transiently amplifying neural progenitors derived from type 1 cells. These progenitors express nestin but lack GFAP and are divided into 2 subgroups, namely, type 2a and type $2 \mathrm{~b}$, based on the presence or absence of the microtubule-associated phosphoprotein doublecortin (Dcx) (Filippov et al., 2003; Fukuda et al., 2003). These cells generate migratory neuroblasts (type 3, expressing Dcx) that rapidly proliferate and exit the cell cycle before maturing into granule neurons (Kronenberg et al., 2003). We observed in TgN-Tau ON mice a significant decrease of the whole type $2 \mathrm{a} / 2 \mathrm{~b}$ population $\left(\mathrm{BrdU}^{+} /\right.$nestin $\left.^{+} / \mathrm{GFAP}^{-}\right)$of 1 - to 5 -day-old progenitor cells ( $\sim 50 \%$; $p=0.0005)$ (Fig. $2 \mathrm{E}$ ) and of type $2 \mathrm{~b}$ progenitor cells $\left(\mathrm{BrdU}^{+} /\right.$nestin $\left.^{+} / \mathrm{Dcx}^{+}\right)(\sim 45 \%$; $p<0.0035)$, concomitant with the overall decrease of the nestin-positive cell population (Fig. 2F). The number of $\mathrm{BrdU}^{+} /$nestin $^{-} / \mathrm{Dcx}^{+}$cells and of early postmitotic neurons was also significantly reduced in TgN-tau ON mice, compared to TgN-tau OFF ( $\sim 24 \% ; p=0.039$, and $\sim 28 \% ; p=0.05$, respectively) (Fig. 2G-J).

\subsection{Reduced long-term survival of new neurons in $\operatorname{TgN}$-tau ON mice}

We next investigated long-term changes in neurogenesis by analyzing cell fate and phenotype of newly born cells that were unaffected by $\mathrm{N}$-tau expression. BrdU was administered for 5 consecutive days from P60 onward, and labeled cells were quantified 4 weeks later, a range of time after which most newly born cells express NeuN (Kempermann et al., 2003). We observed a significant reduction of the total number of 4-week-old cells in TgN-tau ON mice ( $\sim 62 \% ; p=0.001$ ) (Fig. $2 \mathrm{~K}$ ). In parallel, we determined survival rate. This was significantly reduced in TgN-tau ON mice (31.23 \pm 3.22 vs. $53.48 \pm 4.36 ; p=0.0004$ ) (Fig. $2 \mathrm{~L}$ ), implying decreased survival of newly born cells in the TgN-tau ON mice.

A summary of the effects of the expression of N-tau on new progenitor cells and post-mitotic neurons is provided in Fig. 2M.

\subsection{Reduced neurogenesis is not linked to increased caspase- 3-mediated apoptosis}

In the adult DG, newborn cells that are not recruited into functional networks are eliminated by caspase-3-mediated apoptotic cell death in the first 1 to 4 days of life (Biebl et al., 2000), during the transition from amplifying neuroprogenitors to neuroblasts (Kuhn et al., 2005). To analyze whether the reduction in neurogenesis observed in TgN-tau ON mice was due to apoptosis, we performed immunostaining for activated caspase-3. We found a slight reduction of caspase-3-positive cells, but this decrease did not reach statistical significance, in the SGZ of TgN-tau ON mice, compared to TgN-tau OFF (Fig. 3A). However, by TUNEL assay, which detects DNA fragmentation induced by different types of cell death (GraslKraupp et al., 1995), we found that the number of TUNEL-positive cells was significantly increased in TgN-tau ON mice ( $\sim 52 \% ; p=$ 0.03 ) (Fig. 3B). Interestingly, in TgN-tau-ON mice, approximately $40 \%$ of TUNEL-positive cells showed a diffuse cytoplasmic TUNEL labeling that is suggestive of necrosis (Ünal-Cevik et al., 2004) (Fig. 3C), as also confirmed by the appearance of other morphological features of necrosis in nestin-positive cells expressing $\mathrm{N}$-tau (Fig. S3). Moreover, staining for ionized calcium-binding adaptor molecule 1 (Iba-1), which labels microglia, showed that Ntau-induced impaired neurogenesis was accompanied by an increase in microglia population in DG of TgN-tau ON mice $(\sim 35 \%$, $p=0.05$ ) (Fig. 3D).

These alterations were not associated either with changes in the volume of the DG or the whole hippocampus or with changes in the total number of granule cells (Fig. S4).

\subsection{Increased dendritic arbor and spine density of new neurons in TgN-tau ON mice}

As a further step, we investigated whether the integration of new neurons into the existing network was impaired in $\mathrm{N}$-tau transgenic mice. With this aim, mice were injected with retrovirus transducing GFP $\left(10^{8} \mathrm{pfu} / \mathrm{mL}\right.$ ) (Zhao et al., 2006), through stereotaxic delivery into the DG, at P95 (1 month after the activation of the transgene), according to the same time schedule followed for 
A

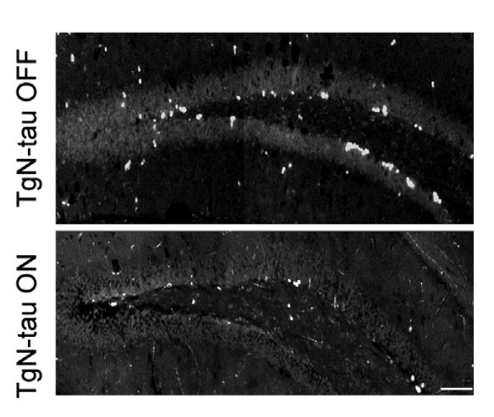

E

$\mathrm{BrdU}^{+} /$nestin $^{+} / \mathrm{GFAP}^{-}$ cells

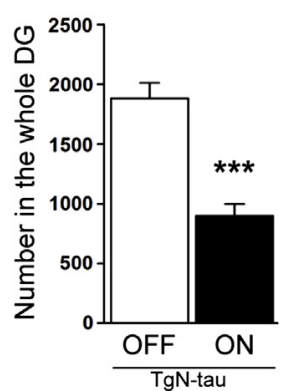

I

$\mathrm{BrdU}^{+} / \mathrm{NeuN}^{+}$cells (new neurons)

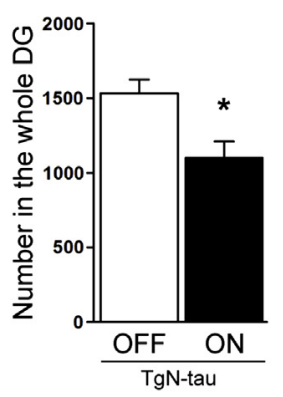

B $\mathrm{BrdU}^{+}$cells

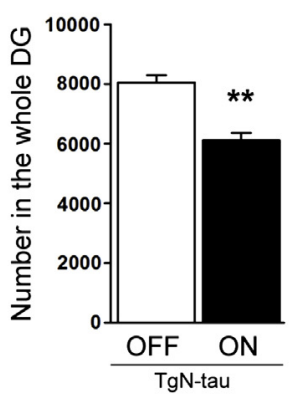

F

nestin $^{+}$cells

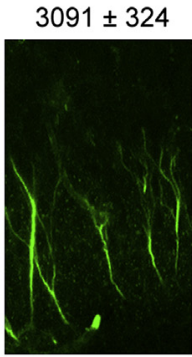

$1524 \pm 170 * * *$

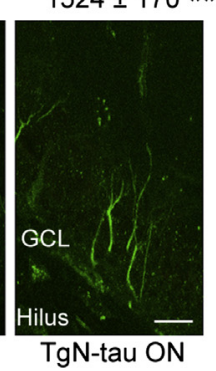

K
C

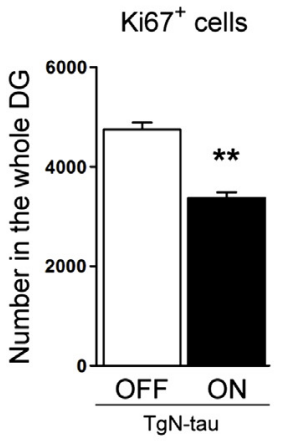

G

$\mathrm{BrdU}^{+} /$nestin $^{-} / \mathrm{Dcx}^{+}$ cells

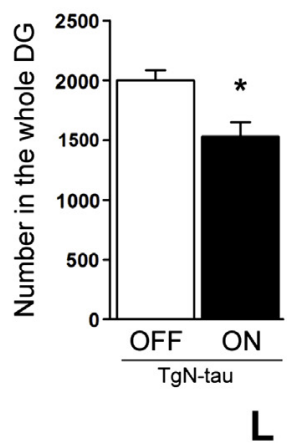

H

$\mathrm{BrdU}^{+} / \mathrm{Dcx}^{+} / \mathrm{NeuN}^{+}$ cells (new neurons)



J

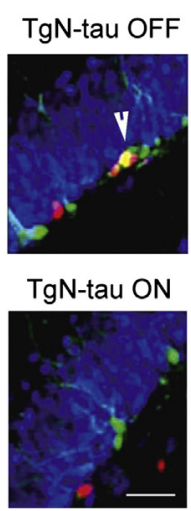

$\mathrm{BrdU}^{+} / \mathrm{NeuN}^{+}$cells

(new neurons 4-wk-old)

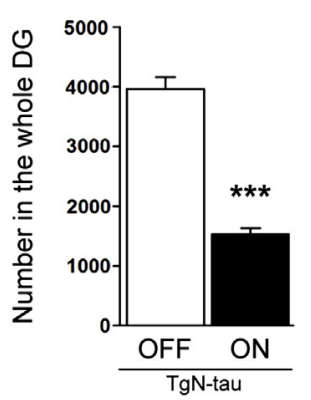

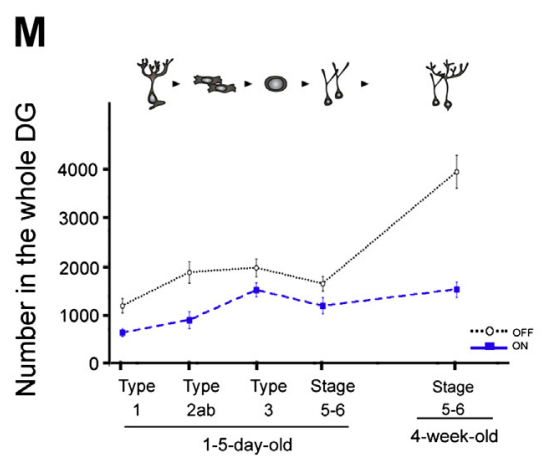

Fig. 2. Decreased neurogenesis in the adult dentate gyrus (DG) of TgN-tau ON mice. (A) Illustration of 1- to 5-day-old newborn cells visualized by BrdU staining. Scale bar, $100 \mu \mathrm{m}$. (B and C) Quantification of BrdU-postive cells and Ki67-positive cells in DG revealed a significant decrease in neurogenesis in TgN-tau ON mice compared to TgN-tau OFF mice. New 1 to 5-day-old stem and progenitor cells, as well as new post-mitotic neurons, were detected by incorporation of BrdU after 5 daily injections in TgN-tau OFF and TgN-tau ON mice (the latter having the transgene active), and classified based on the expression of specific markers. (D) The number of putative stem cells (type 1; BrdU ${ }^{+} /$nestin $^{+} / \mathrm{GFAP}^{+}$) decreased by $\sim 50 \%$ in TgN-tau ON mice. (E) The activation of the $\mathrm{N}$-tau transgene also reduced by $\sim 50 \%$ the number of putative type 2 a progenitor cells $\left(\mathrm{BrdU}^{+} /\right.$nestin $\left.^{+} / \mathrm{GFAP}^{-}\right)$, as well as (F) the total number of nestin-positive cells (the latter were immunolabeled with chicken polyclonal antibody against nestin [PA1-46338 1:1000; Thermo-Fisher Pierce]; scale bar, $20 \mu \mathrm{m}$ ) and $(\mathrm{G})$ that of type 3 progenitor cells $\left(\mathrm{BrdU}^{+} /\right.$nestin $\left.^{-} / \mathrm{Dcx}^{+}\right)$. (H) The differentiating early post-mitotic neurons at stage $5\left(\mathrm{BrdU}^{+} / \mathrm{Dcx}^{+} / \mathrm{Neun}^{+}\right)$and $(\mathrm{I})$ stage $6\left(\mathrm{BrdU}^{+} / \mathrm{Neun}^{+}\right)$were significantly decreased in TgN-tau ON mice. (J) Representative confocal images of new 1- to 5-day-old DG neurons labeled by BrdU/Dcx/NeuN-positive (red, green, and blue, 
the immunohistochemical analyses. We focused on dendritic length, dendritic arborization and spine density at 10 and $30 \mathrm{dpi}$, the latter being a time point at which neuronal cell death was not yet apparent.

The analysis of GFP-positive neurons at 10 dpi revealed a significantly decreased dendritic length in TgN-tau ON mice compared to TgN-tau OFF (Fig. 4A and B). This difference was not observed at $30 \mathrm{dpi}$, when the dendritic length of new neurons was similar in TgN-tau ON and TgN-tau OFF mice (Fig. 4A and B). The number of branching points in GFP-positive neurons at $10 \mathrm{dpi}$ was significantly lower in TgN-tau ON mice than in TgN-tau OFF. This difference, however, reversed at $30 \mathrm{dpi}$, when the $\mathrm{TgN}$-tau ON exhibited a higher number of branching points (Fig. 4A-C). Normally, spine growth starts in the adult-generated DG neurons at approximately $16 \mathrm{dpi}$ and reaches a plateau at $56 \mathrm{dpi}$; however, at $28 \mathrm{dpi}$, the exponential phase of growth has already ceased, and this stage can be considered representative of the achievement of the neuronal morphological maturity (Zhao et al., 2006). Quantification of spine density in the DG neurons at 30 dpi revealed a significant increase in TgN-tau ON mice, compared to TgN-tau OFF (Fig. 4D and E).

Altogether, these morphological analyses point to an increase in dendritic arborization and spine density occurring, in TgN-tau ON surviving neurons, during the developmental stages between 10 and 30 dpi.

\subsection{Increased anxiety-related behavior in $\mathrm{TgN}$-tau ON mice}

TgN-tau ON and TgN-tau OFF mice, as well as control groups (nestin-rtTA and WT mice), were preliminarily tested for basal behaviors in an open field, with no differences between groups in any of the variables taken into consideration (Figs. S5 and S6). Anxiety-related behaviors were evaluated in an elevated plus maze. Toward this aim, 2 different experimental settings were prepared, and independent groups of mice were assigned to 1 setting or the other for subsequent testing. A dimly lit, silent room housed the apparatus for the non-threatening condition, which was successively turned into a brightly lit one, with direct lighting of the apparatus and moderate background noise, for the fearful condition. This procedure is useful in discriminating the relative contribution of fear of external threats, on 1 hand, and the strength of novelty-seeking behavior, on the other, to the performance of the animals in the maze (Revest et al., 2009; Saab et al., 2009), thus reducing the risk of misinterpreting a possible weakening in their attitude toward explore novel environments as increased susceptibility to anxiety. Indeed, TgN-tau ON $(\mathrm{n}=8)$ and TgN-tau OFF $(\mathrm{n}=$ 10) mice showed comparable tendency to visit the open arms of the apparatus when tested in non-threatening conditions (Fig. 5A and $\mathrm{B} ; p>0.454$; Student $t$ test); however, a statistically significant difference between groups emerged during testing in the fearful environment, with TgN-tau ON mice $(\mathrm{n}=9)$ entering and exploring the open arms less than TgN-tau OFF mice $(n=11)$ (Fig. $5 \mathrm{C}$ and $\mathrm{D}$; $p<0.05$; Student $t$ test). Such a tendency to show increased anxiety-related responses in threatening conditions was confirmed by successive testing of the animals in the light/dark emergence test. Here, TgN-tau ON $(n=8)$ and TgN-tau OFF $(n=10)$ mice were allowed to explore for 10 minutes an open field bathed in direct light and were provided with a dark plastic cylinder, placed lengthwise along a wall, that was useful for the animals to shelter.
Again, a statistically significant difference was observed between groups with regard to the number of entries into the cylinder and the time spent in the cylinder, with TgN-tau ON mice hiding in the cylinder more often, and for longer periods, than TgN-tau OFF mice (Fig. 5E and F; $p<0.05$; Student $t$ test). Conversely, TgN-tau ON mice were less inclined to explore the open field compared to TgNtau OFF mice, as indicated by the significantly shorter distance that they traveled in the lighted area of the apparatus (Fig. 5G; $p<0.05$; Student $t$ test). Depression-related behaviors were also assessed in TgN-tau ON mice, with no differences observed in comparison with TgN-tau OFF mice (Fig. S7). No significant differences between TgNtau OFF, nestin-rtTA and WT mice were observed in anxiety- and depression-related behaviors, which rules out nonspecific effects exerted by the genetic manipulation on the behavioral responses of transgenic mice (Fig. S8).

\subsection{Impaired episodic-like memory in $\mathrm{TgN}$-tau ON mice}

Learning and memory abilities of TgN-tau mice were first addressed by testing in a reference version of the Morris Water Maze. After being trained to a visible platform for 2 days, to exclude motor deficits or changes in visual acuity, $\operatorname{TgN}$-tau ON $(n=9)$ and TgN-tau OFF ( $\mathrm{n}=11)$ mice were subjected to 6 daily training sessions, with 4 trials per day, during which animals from both groups learned reliably to navigate to a hidden platform in the pool by using distal cues, with comparable rate (Fig. 6A; effect of group: $\mathrm{F}_{1,18}=0.46, p=0.506$; effect of training: $\mathrm{F}_{5,90}=20.43, p<0.001$; training $\times$ group interaction: $\mathrm{F}_{5,90}=0.51, p=0.768 ; 2$-way repeated-measures analysis of variance [ANOVA]). Long-term retention of the memory for the platform position was further assessed by a probe test, carried out 24 hours after the completion of the last training session, with animals allowed to swim in the pool for 60 seconds in the absence of the platform. As normally observed, both TgN-tau ON and Tg-tau OFF mice spent more time in the sector of the pool where the platform was located during training, compared to the other sectors (Fig. 6B; effect of group: $\mathrm{F}_{1,18}=1.69, p=0.210$; effect of sector: $\mathrm{F}_{3,54}=18.27, p<0.001$; sector $\times$ group interaction: $F_{3,54}=0.61, p=0.610 ; 2$-way repeatedmeasures ANOVA), showing a negligible amount of thigmotaxis (Fig. 6C; $p=0.571$; Student $t$ test).

Independent groups of TgN-tau ON $(\mathrm{n}=10)$ and TgN-tau OFF $(\mathrm{n}=12)$ mice were tested in a second water maze protocol, which addresses rodents' ability to perform 1-trial learning and episodiclike memory (Chen et al., 2000; Farioli-Vecchioli et al., 2012; Zeng et al., 2001). In this delayed matching-to-place version of the task, mice are confronted with a series of successive platform locations and trained to navigate to each of them until reaching a rigorous performance criterion (or a maximal number of trials), after which the platform is moved to a different location in the pool and a new training session starts. In such a procedure, the earlier locations of the platform, which are encoded in long-term memory, are likely to cause interference when the most recently encoded location has to be selectively retrieved, to complete the current session successfully. Thus, for each training session except the first, a decrease of escape latency in the second trial compared to the first trial points to the ability of the mice to rapidly acquire the memory for the new platform location, avoiding the interference exerted by the memories previously acquired for the other locations. As training proceeded, both TgN-tau ON and TgN-tau OFF mice

respectively) in TgN-tau ON and OFF mice. The merge shows neurons positive for all 3 antigens as yellow cells present in TgN-tau ON, indicated by white arrowhead. Scale bar, $25 \mu \mathrm{m}$. (K) In TgN-tau ON mice, the total number of new terminally differentiated neurons (4-week-old, $\mathrm{BrdU}^{+} / \mathrm{NeuN}^{+}$) was significantly reduced. (L) Expression of $\mathrm{N}$-tau resulted also in reduced long-term survival of newborn cells. Survival was quantified as the ratio of the number of 4-week-old BrdU ${ }^{+}$cells to that of 1 - to 5-day-old BrdU ${ }^{+}$cells. (M) Summary, from data in Fig. 2, of the effect of the activation of N-tau on new progenitor cells and post-mitotic neurons. Results (B-I and $\mathrm{K}-\mathrm{L}$ ) are derived from analysis of at least 3 animals per group and are presented as mean \pm SEM. ${ }^{* *} p<0.01,{ }^{*} p<0.05$, Student $t$ test versus TgN-tau OFF. 
A

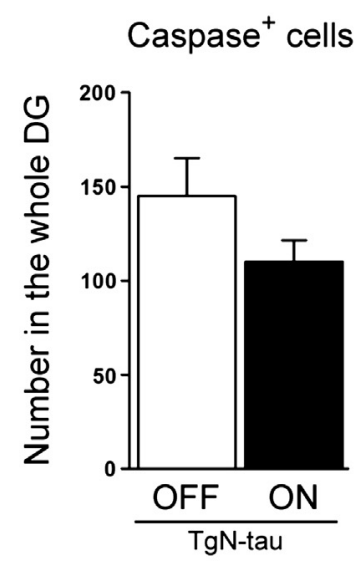

\section{B}

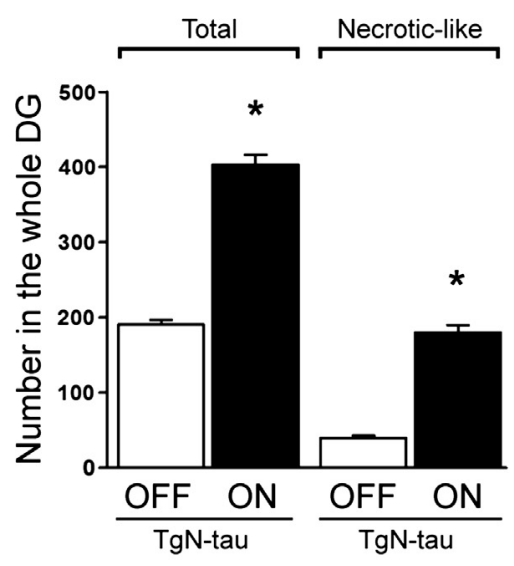

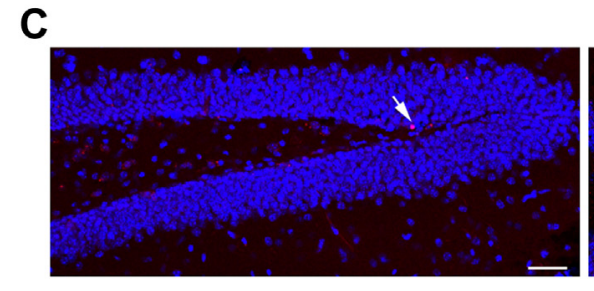

TgN-tau OFF

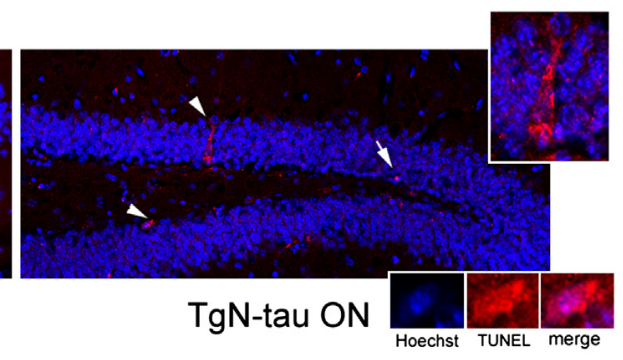

D

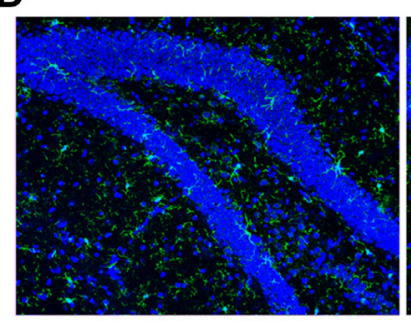

TgN-tau OFF

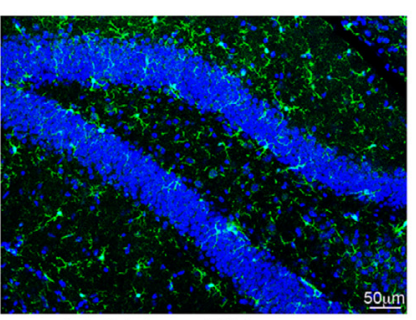

TgN-tau ON

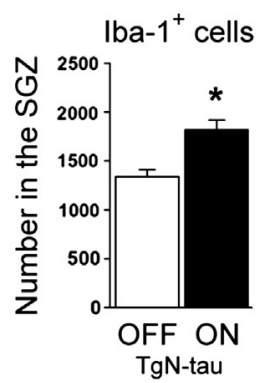

Fig. 3. Analysis of cell death in TgN-tau mice. (A) A slight difference between TgN-tau ON and TgN-tau OFF mice was observed in the number of caspase-3-positive new neurons at P95, but this decrease did not reach statistical significance. (B) TUNEL assay detected a significantly increased number of dead or dying neurons. (C) In TgN-tau ON mice, the majority of TUNEL-positive cells displayed diffuse TUNEL staining (arrowheads), which is suggestive of a necrotic process. Insets show higher magnification of the TUNEL staining in the 2 cells marked by arrowheads. Arrows indicate classical TUNEL staining inside nucleus. (D) Immunostaining and quantification of the number of Iba-1-positive cells in the subgranular zone (SGZ). Results are derived from analysis of at least 3 animals per group and are presented as mean \pm SEM. ${ }^{*} p<0.05$, Student $t$ test versus TgN-tau OFF.

retained their ability to locate reliably the hidden platform at each of the 5 positions to which it was sequentially moved (Fig. 6D, left to right; effect of trial, for all positions: $\mathrm{F}_{4,80}>5.60, p<0.001$; trial $\times$ group interaction, for all positions: $\mathrm{F}_{4,80}<0.50, p>0.303 ; 2$-way repeated-measures ANOVA). However, although no effect of group was detected for the first and the second platform positions (Fig. 6D, positions \#1 and \#2; effect of group, for both positions: $\mathrm{F}_{1,20}<2.73, p>0.095$ ), a significant difference between groups emerged for the third, fourth, and fifth positions, with TgN-tau ON mice reducing their escape latencies at a slower rate compared to TgN-tau OFF (Fig. 6D, positions 3, 4, and 5; effect of group, for all positions: $F_{1,20}>9.99, p<0.049$ ). Consistently, a significant difference between genotypes also emerged with regard to the reduction of escape latencies animals achieved as they passed from the first to the second trial of the last 2 training sessions (Fig. 6E; $p<$ 0.01 ; Student $t$ test). These data suggest that $\mathrm{TgN}$-tau mice began to fail in rapidly encoding new spatial memories during the latter stages of training, when they had a greater amount of previously stored spatial information to deal with, to avoid interference. This is further reflected by the significantly higher number of trials that
TgN-tau ON mice needed to reach the performance criterion over the last 2 training sessions, compared to TgN-tau OFF (Fig. 6F; $p<$ 0.01; Student $t$ test). None of control groups (nestin-rtTA and WT mice) differed from TgN-tau OFF mice when tested in the delayed matching-to-place version of the water maze (Fig. S9).

\section{Discussion}

Here we present a murine model that is, to our knowledge, the first specifically aimed to analyze the direct effects of the overexpression of a pathological tau species on adult hippocampal neurogenesis.

Our model is based on the selective and conditional expression in adult neural precursor cells of the N-terminal-26-230 fragment of the longest human tau isoform (Canu et al., 1998) using the TetOn system under the control of nestin promoter.

The activation of the transgene resulted in the expression of $\mathrm{N}$ tau protein in nestin-positive cells of the DG and SVZ, where neural precursors are present. This led to a drastic reduction in the number of nestin-positive cells, that is, of type 1 putative stem cells and type 

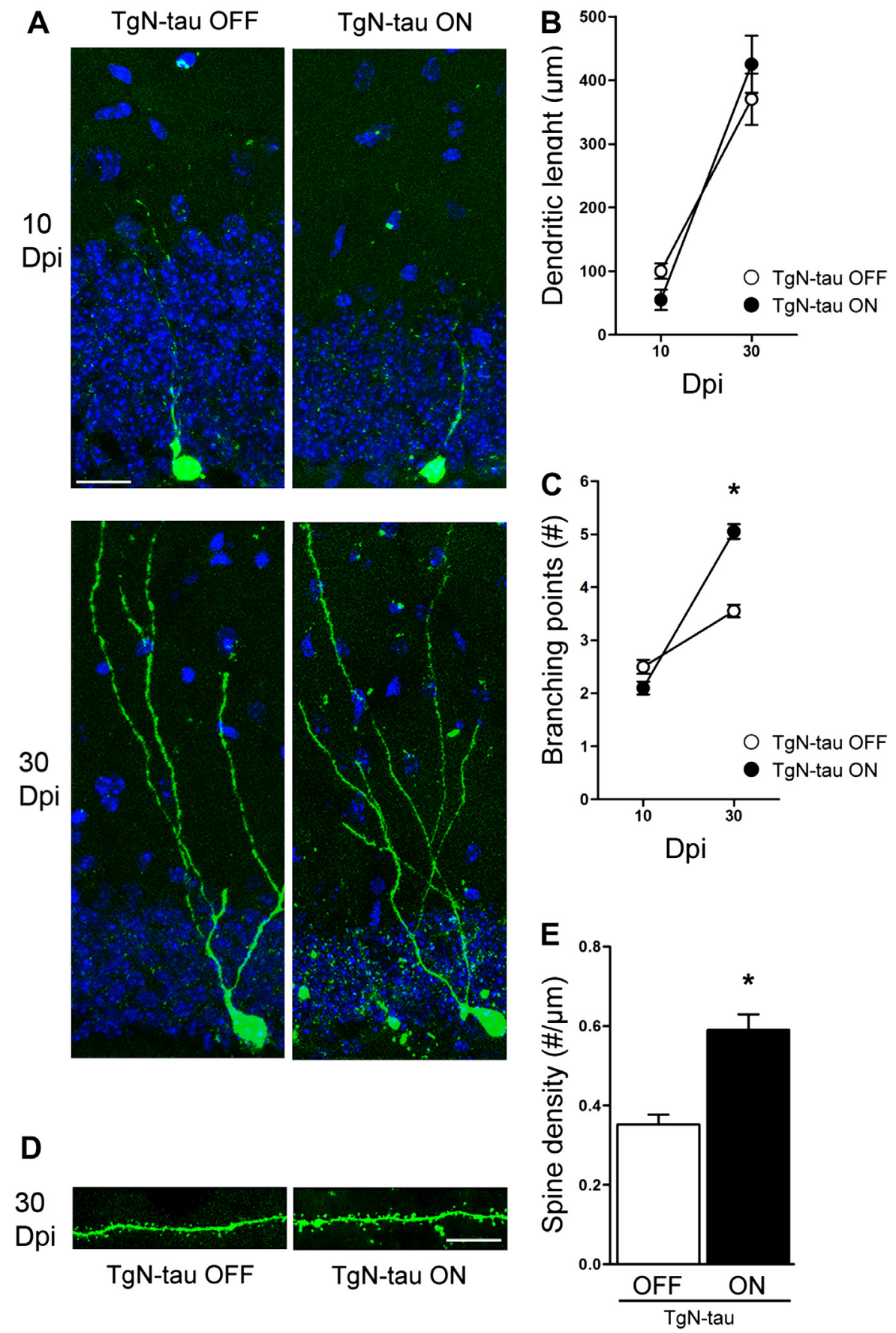

Fig. 4. Morphological analysis of the development of adult-generated dentate gyrus (DG) neurons. (A) Representative morphology of adult-generated neurons labeled with GFP by retrovirus-mediated gene transduction, analyzed at 10 and $30 \mathrm{dpi}$ in TgN-tau mice (in TgN-tau ON mice, the N-tau transgene was activated 90 days before the infection). GFP-positive neurons (green) are localized at the hilar border of the DG, the cells of which are identified by Hoechst 33258 staining (blue). Molecular layer is localized at the upper side of the images. Scale bar, $150 \mu \mathrm{m}$. (B) Quantification of the dendritic length of adult-generated GFP-positive neurons at 10 and $30 \mathrm{dpi}$. (C) Quantification of branching points at 10 and 30 dpi. (D) Quantification of spine density in dendritic processes of GFP-positive neurons at $30 \mathrm{dpi}$. (E) Representative confocal images of dendrites and spines GFP-positive DG neurons at $30 \mathrm{dpi}$. Scale bar, $12 \mu \mathrm{m}$. Data in B, C, and E are presented as mean \pm SEM. $^{*} p<0.05$ versus TgN-tau OFF, Student $t$ test. Results are derived from the analysis of at least 3 animals per group.

2a,b progenitor cells, accompanied by a lesser decrease of type 3 and 1- to 5-day-old terminally differentiated postmitotic cells. The latter finding is likely to be ascribed to the physiological inactivation of nestin promoter, which ceases to drive the expression of $\mathrm{N}$-tau in these neuronal cells. Moreover, the total number of Ki67positive cells, long-term survival, and neurogenesis were strongly reduced, albeit not totally blocked, in the DG of $\mathrm{TgN}$-tau ON mice. Indeed, a residual level of neurogenesis persisted several months after the induction of $\mathrm{N}$-tau expression. Also, we found that Doxtreatment reduced cell proliferation in the SVZ of TgN-tau ON mice, consistent with the expression of $\mathrm{N}$-tau in the second main neurogenic niche of the brain (Fig. S1).

The observed effects on neurogenesis in TgN-tau ON mice were linked to the finding that the number of TUNEL-positive cells was significantly increased, suggesting that $\mathrm{N}$-tau expression leads to cell death. Indeed, we found that nestin-positive cells expressing 

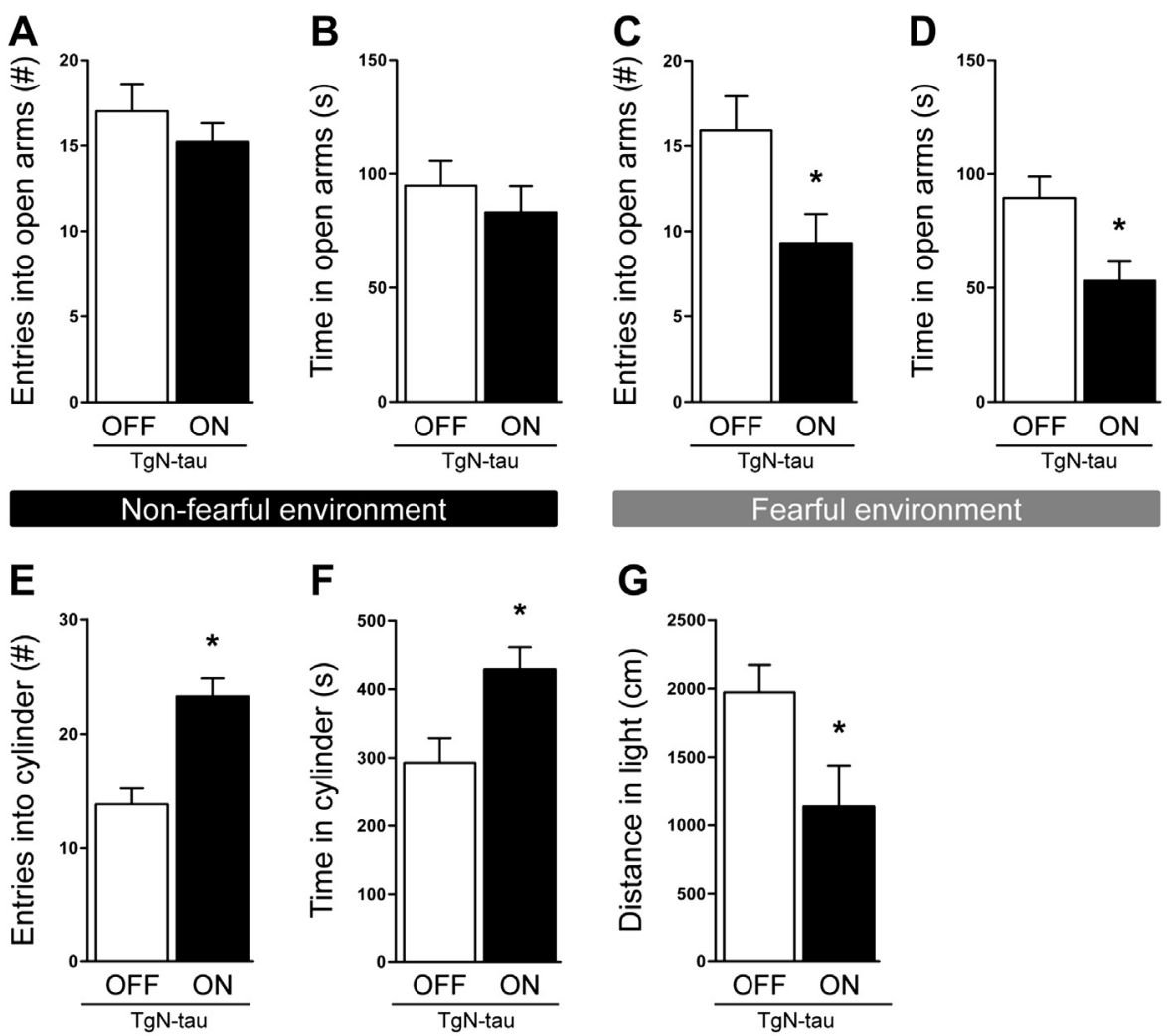

F

\section{G}

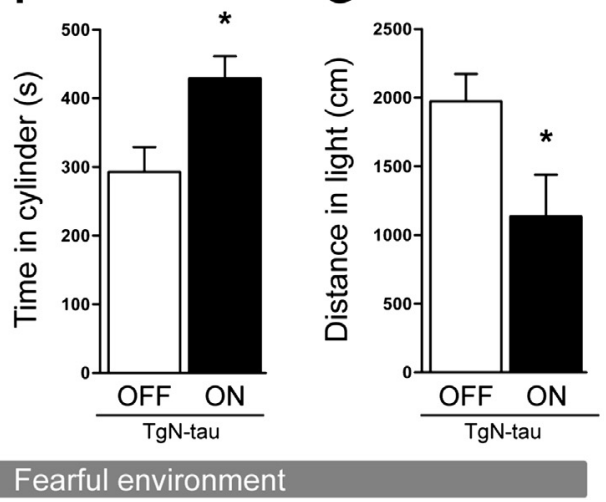

Fig. 5. Increased anxiety-related behaviors in TgN-tau mice. (A and B) In the elevated plus maze test, TgN-tau ON and TgN-tau OFF mice showed a comparable tendency to enter and explore the open arms of the apparatus, when the latter was placed in a dimly lit, non-threatening environment. (C and D) Conversely, a statistically significant difference between the 2 groups was found in both the number of entries into the open arms and the time spent in the open arms, when the mice were tested in a fearful environment, with direct lighting of the apparatus and moderate background noise. $(\mathrm{E}-\mathrm{G}$ ) Increased anxiety responses in threatening conditions were confirmed in the light/dark emergence test, where TgNtau ON mice showed a significantly higher number of entries into a dark cylinder suitable for providing protection, and spent a significantly greater amount of time inside the cylinder, compared to TgN-tau OFF mice. Conversely, TgN-tau ON mice were less inclined to explore the lighted area of the apparatus, compared to TgN-tau OFF mice, as indicated by the significantly shorter distance that they traveled out of the cylinder. Results are presented as mean \pm SEM. ${ }^{*} p<0.05$.

$\mathrm{N}$-tau showed signs of degeneration reminiscent of necrosis (Fig. S3A and B) (Barros et al., 2003). Moreover, the finding that the number of activated caspase-3-positive cells was not increased in TgN-tau ON mice, compared to controls, and that many of the dying cells showed a diffused TUNEL staining, are further arguments in support of a non-apoptotic cell death pathway activated by N-tau. These findings are consistent with our previous report showing that, in vitro, the same tau fragment caused a caspase3-independent, NMDAR-mediated, cell death (Amadoro et al., 2006). Because type 1 and type 2a precursor cells express NMDAR1 and NMDAR2B receptors that are likely extrasynaptic (Nácher et al., 2007), it is reasonable to think that N-tau might impair adult neurogenesis through the activation of these receptors. It is known that the NR2B receptor negatively regulates adult hippocampal neurogenesis and spatial memory (Hu et al., 2008). Moreover, nitric oxide production after the NMDAR activation blocks the proliferation of neuronal precursors. Potential downstream effectors of nitric oxide include the second messenger cyclic GMP, as well as p53, p21, and phospho-Retinoblastoma (pRb) proteins. (Gibbs, 2003). Rb, which is expressed mainly in proliferating precursors (Okano et al., 1993), is hyperphosphorylated and then inactive in several tauopathies (Stone et al., 2011).

Alternatively, $\mathrm{N}$-tau may indirectly control $\mathrm{Rb}$ function through the adaptor protein Che-1, which has been reported to inhibit $\mathrm{Rb}$ suppression activity by interfering with the recruitment of HDAC1 by $\mathrm{Rb}$ (Bruno et al., 2002). Because the interaction between $\mathrm{N}$-tau and Che-1 is modulated during neuronal apoptosis, when the reactivation of aberrant cell cycle leads to neuronal death (Barbato et al., 2003), it is likely that expression of N-tau in neuronal precursors may perturb the interplay between $\mathrm{Rb} / \mathrm{Che}-1$ in the regular control of cell cycle. Thus, N-tau, like other pathological tau species, may induce $\mathrm{Rb}$ inactivation in neuronal precursor cells (Lee et al., 1994; Stone et al., 2011), which in turn raises conflict between ongoing proliferative and proliferation-inhibitory stimuli leading to cell death, as commonly observed after ablation of inhibitors of proliferation such as, for instance, the Btg-1 protein (FarioliVecchioli et al., 2012).

Further studies are needed to ascertain the mechanism(s) underlying the process of cell death associated with $\mathrm{N}$-tau expression in neuronal precursor cells.

A potential concomitant consequence of $\mathrm{N}$-tau-induced cell death was the increased microglia cell recruitment in the SGZ of TgN-tau ON mice, suggesting that $\mathrm{N}$-tau-induced impairment of neurogenesis caused a mild inflammatory response. Although microglia could have an important regulatory role at the early stages of neurogenesis by rapidly removing cell debris (Sierra et al., 2010), the phagocytosis could become detrimental for the surrounding newborn neurons by further inducing neuronal death (Neher et al., 2011). Thus microglia could exacerbate, in turn, Ntau-induced negative effects on neurogenesis, extending the impact of $\mathrm{N}$-tau overexpression also to neuronal precursor cells in which the nestin promoter is inactive. However, we cannot exclude a direct action of $\mathrm{N}$-tau on type 3 cells (nestin ${ }^{-} / \mathrm{Dcx}^{+}$), given that the half-life of tau (60 hours and more in its phosphorylated form) 


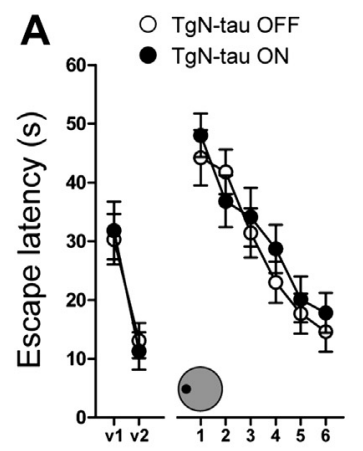

Training session

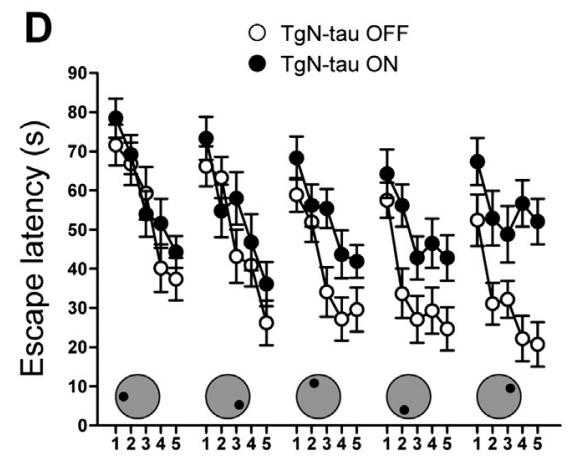

Training trial

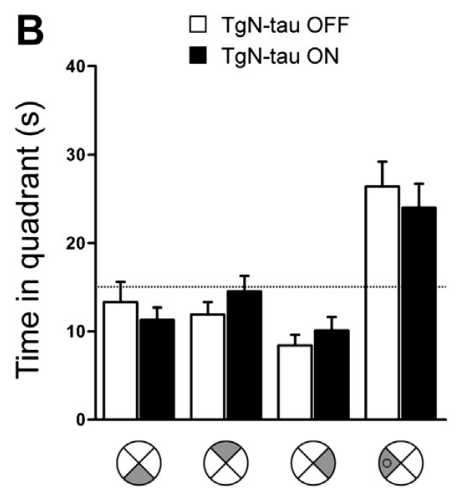

E

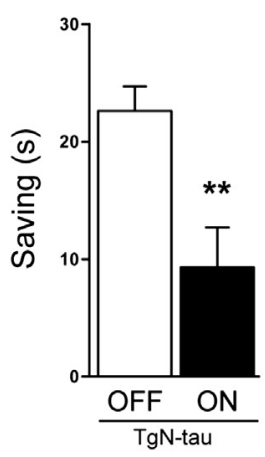

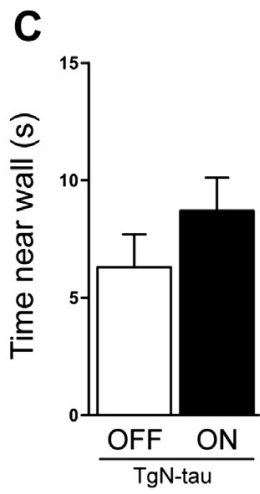

$\mathbf{F}$

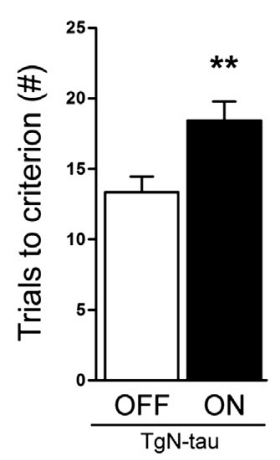

Fig. 6. Learning and memory abilities of TgN-tau mice. (A) After being pre-trained to a visible platform for 2 days (v1-v2), to exclude motor deficits or weakening in visual acuity, both TgN-tau ON and TgN-tau OFF mice learned reliably to navigate to a hidden platform in a reference version of the Morris Water Maze, as indicated by the significant decrease of escape latencies over the 6 days of training. (B) On a probe test, performed 24 hours after the completion of the last training session, both groups of mice spent a significantly greater amount of time in the target quadrant of the pool, where the platform was located during training, compared to the other quadrants. (C) Both groups of mice showed negligible thigmotaxis (i.e., swimming in a circular manner along the wall of the pool) during the probe test. (D) In a delayed matching-to-place version of the Morris Water Maze, TgN-tau ON mice learned the new platform position at a significantly slower rate compared to TgN-tau OFF mice over the third to fifth training sessions, which indicates a failure in the rapid encoding of new spatial memories when previously acquired spatial information is likely to exert interference. (E) Reduction of escape latencies (saving) achieved by mice as they passed from the first to the second trial of the fourth and fifth training sessions (averaged). (F) Number of trials that mice needed to reach the performance criterion ( 3 successive trials with mean escape latency $<20$ seconds) in the fourth and fifth training sessions (averaged). Results are presented as mean \pm SEM. ${ }^{* *} p<0.01$.

(Poppek et al., 2006) may allow $\mathrm{N}$-tau expression to persist in some of these cells.

The expression of human tau-4R has been reported to suppress proliferation and to promote neuronal differentiation in the DG of tau knockin/knockout mice (Sennvik et al., 2007). Although we found that surviving new neurons in TgN-tau ON mice showed an increase in dendritic complexity and spine density, we assume that this phenotype is not the consequence of the differentiation promoted by $\mathrm{N}$-tau. This assumption is based on the finding that a similar tau fragment suppressed nerve growth factor-induced neurite extension in PC12 cell line (Brandt et al., 1995) or in primary embryonic hippocampal cultures when $\mathrm{N}$-tau was overexpressed (N. Canu, unpublished data, 2012); rather, we favor the hypothesis that this phenotype may represent a neural compensation (Kolb Brown et al., 2001) to the effects triggered by $\mathrm{N}$-tau expression.

Although a large body of data collected in recent years suggests a role for adult neurogenesis in some kinds of hippocampus-dependent memory, the presence of conflicting results and inconsistencies in the literature has made it difficult to identify the basic operating principles of adult-born neurons in learning and memory processes (Deng et al., 2010). However, both computational and behavioral approaches seem to indicate that adult neurogenesis could ultimately contribute to enhancing the extent of information encoded by the DG and improving pattern separation, which refers to the ability to discriminate among potentially overlapping experiences (Aimone et al., 2011; Sahay et al., 2011) and has been recognized as intrinsic to episodic memory (Yassa and Stark, 2011). Consistently, the loss of newborn neurons in the DG of $\mathrm{TgN}$-tau ON mice was associated with a selective failure in a delayed matching-to-place version of the Morris Water Maze, in a test specifically designed to assess episodic-like components of memory (Chen et al., 2000; Zeng et al., 2001). In addition, an increased anxiety-related behavior was observed in TgN-tau ON mice when tested under stressful conditions, which adds to previous observations indicating a potential role for adult neurogenesis in the regulation of emotional responses (Revest et al., 2009; Bergami et al., 2008). It is noteworthy that both anxiety and a selective weakening of episodic memory have been documented at the early onset of Alzheimer's disease (Gallagher and Koh, 2011).

In conclusion, although the model used presents methodological limits (i.e., lack of comparison between the effects of $\mathrm{N}$-tau versus full-length tau overexpression that would better clarify the specific role of $\mathrm{N}$-tau), our newly developed conditional murine model allowed us to demonstrate that overexpression of a tau modification, which is associated with disease, may directly affect adult DG neurogenesis and episodic-like memory. We believe that our work provides new insights into the complex mechanisms underlying impairment of hippocampal neurogenesis in tau-associated neurodegenerative disease.

As a next step, it would be interesting to clarify by which mechanisms nestin-positive neuronal precursors could eventually express $\mathrm{N}$-tau or other pathological tau species. Existing data suggest that tau secretion, followed by cell-to-cell spreading, might 
underlie the ability of adult hippocampal neuronal precursor cells to take up and express pathological tau species (Clavaguera et al., 2009; Saman et al., 2012). Such a mechanism bears major implications also for the effectiveness of neuronal stem cell therapy as a potential treatment for Alzheimer's disease.

\section{Disclosure statement}

The authors declare no potential conflicts of interest.

\section{Acknowledgements}

We thank F.H. Gage for providing the CAG-GFP viral vector, L. Micheli for suggestions in the retrovirus production, and A. Graziani for mouse tail biopsy. This work was supported by grants from Italian Ministry of Education, University and Research (PRIN 2009, 2009KP83CR-02; 2009KP83CR-03; 2009KP83CR-01 to N.C., E.M., and V.C., respectively) and from the Italian Ministry of Economy (Project FaReBio; to F.T.). D.S. was supported by the Guardia di Finanza-Italian Ministry of Economy.

\section{Appendix A. Supplementary data}

Supplementary data associated with this article can be found, in the online version, at 10.1016/j.neurobiolaging.2013.05.010.

\section{References}

Aimone, J.B., Deng, W., Gage, F.H., 2011. Resolving new memories: a critical look at the dentate gyrus, adult neurogenesis, and pattern separation. Neuron 70, 589-596.

Amadoro, G., Ciotti, M.T., Costanzi, M., Cestari, V., Calissano, P., Canu, N., 2006. NMDA receptor mediates tau-induced neurotoxicity by calpain and ERK/MAPK activation. Proc. Natl. Acad. Sci. U.S.A. 103, 2892-2897.

Amadoro, G., Corsetti, V., Atlante, A., Florenzano, F., Capsoni, S., Bussani, R., Mercanti, D., Calissano, P., 2012. Interaction between $\mathrm{NH}(2)$-tau fragment and $A \beta$ in Alzheimer's disease mitochondria contributes to the synaptic deterioration. Neurobiol. Aging 33, 833.e1-833.e25.

Barbato, C., Corbi, N., Canu, N., Fanciulli, M., Serafino, A., Ciotti, M., Libri, V., Bruno, T., Amadoro, G., De Angelis, R., Calissano, P., Passananti, C., 2003. Rb binding protein Che-1 interacts with Tau in cerebellar granule neurons. Modulation during neuronal apoptosis. Mol. Cell. Neurosci. 24, 1038-1050.

Barros, L.F., Kanaseki, T., Sabirov, R., Morishima, S., Castro, J., Bittner, C.X., Maeno, E., Ando-Akatsuka, Y., Okada, Y., 2003. Apoptotic and necrotic blebs in epithelial cells display similar neck diameters but different kinase dependency. Cell Death Differ. 10, 687-697.

Bergami, M., Rimondini, R., Santi, S., Blum, R., Götz, M., Canossa, M., 2008. Deletion of TrkB in adult progenitors alters newborn neuron integration into hippocampal circuits and increases anxiety-like behavior. Proc. Natl. Acad. Sci. U.S.A. 105, 15570-15575.

Biebl, M., Cooper, C.M., Winkler, J., Kuhn, H.G., 2000. Analysis of neurogenesis and programmed cell death reveals a self-renewing capacity in the adult rat brain. Neurosci. Lett. 291, 17-20.

Brandt, R., Léger, J., Lee, G., 1995. Interaction of tau with the neural plasma membrane mediated by tau's amino-terminal projection domain. J. Cell Biol. 131, 1327-1340.

Bullmann, T., de Silva, R., Holzer, M., Mori, H., Arendt, T., 2007. Expression of embryonic tau protein isoforms persist during adult neurogenesis in the hippocampus. Hippocampus 17, 98-102.

Bruno, T., De Angelism, R., De Nicola, F., Barbato, C., Di Padova, M., Corbi, N., Libri, V., Benassi, B., Mattei, E., Chersi, A., Soddu, S., Floridi, A., Passananti, C., Fanciulli, M., 2002. Che-1 affects cell growth by interfering with the recruitment of HDAC1 by Rb. Cancer Cell 5, 387-399.

Canu, N., Dus, L., Barbato, C., Ciotti, M.T., Serafino, A., Dus, L., Calissano, P., 1998. Tau cleavage and dephosphorylation in cerebellar granule neurons undergoing apoptosis. J. Neurosci. 18, 7061-7074.

Chatterjee, S., Sang, T.K., Lawless, G.M., Jackson, G.R., 2009. Dissociation of tau toxicity and phosphorylation: role of GSK-3beta, MARK and Cdk5 in a Drosophila model. Hum. Mol. Genet. 18, 164-177.

Chen, G., Chen, K.S., Knox, J., Inglis, J., Bernard, A., Martin, S.J., Justice, A., McConlogue, L., Games, D., Freedman, S.B., Morris, R.G., 2000. A learning deficit related to age and beta-amyloid plaques in a mouse model of Alzheimer's disease. Nature 408, 975-979.

Clavaguera, F., Bolmont, T., Crowter, R.A., Abramowski, D., Frank, S., Probst, A., Fraser, G., Stalder, A.K., Beibel, M., Staufenbiel, M., Jucker, M., Goedert, M.,
Tolnay, 2009. Transmission and spreading of tauopathy in transgenic maouse brain. Nat. Cell Biol. 11, 909-913.

Corsetti, V., Amadoro, G., Gentile, A., Capsoni, S., Ciotti, M.T., Cencioni, M.T. Atlante, A., Canu, N., Rohn, T.T., Cattaneo, A., Calissano, P., 2008. Identification of a caspase-derived $\mathrm{N}$-terminal tau fragment in cellular and animal Alzheimer's disease models. Mol. Cell Neurosci. 38, 381-392.

Deng, W., Aimone, J.B., Gage, F.H., 2010. New neurons and new memories: how does adult hippocampal neurogenesis affect learning and memory? Nat. Rev. Neurosci. $11,339-350$.

Emsley, J., Mitchell, B., Kempermann, G., Macklis, J.D., 2005. Adult neurogenesis and repair of the adult CNS with neural progenitors, precursors, and stem cells. Prog. Neurobiol. 75, 321-341.

Farioli-Vecchioli, S., Saraulli, D., Costanzi, M., Pacioni, S., Cinà, I., Aceti, M., Micheli, L. Bacci, A., Cestari, V., Tirone, F., 2008. The timing of differentiation of adult hippocampal neurons is crucial for spatial memory. PLoS Biol. 6, e246.

Farioli-Vecchioli, S., Micheli, L., Saraulli, D., Ceccarelli, M., Cannas, S., Scardigli, R., Leonardi, L., Cinà, I., Costanzi, M., Ciotti, M.T., Moreira, P., Rouault, J.P., Cestari, V., Tirone, F., 2012. Btg1 is required to maintain the pool of stem and progenitor cells of the dentate gyrus and subventricular zone. Front. Neurosci. 6, 124.

Filippov, V., Kronenberg, G., Pivneva, T., Reuter, K., Steiner, B., Wang, L.P., Yamaguchi, M., Kettenmann, H., Kempermann, G., 2003. Subpopulation of nestin-expressing progenitor cells in the adult murine hippocampus shows electrophysiological and morphological characteristics of astrocytes. Mol. Cell Neurosci. 23, 373-382.

Fuster-Matanzo, A., Llorens-Martín, M., Jurado-Arjona, J., Avila, J., Hernández, F., 2012. Tau Protein and Adult Hippocampal Neurogenesis. Front. Neurosci. 6, 104.

Fukuda, S., Kato, F., Tozuka, Y., Yamaguchi, M., Miyamoto, Y., Hisatsune, T., 2003. Two distinct subpopulations of nestin-positive cells in adult mouse dentate gyrus. J. Neurosci. 23, 9357-9366.

Gaiano, N., Kohtz, J.D., Turnbull, D.H., Fishell, G., 1999. A method for rapid gain-offunction studies in the mouse embryonic nervous system. Nat. Neurosci. 2, 812-819.

Gallagher, M., Koh, M.T., 2011. Episodic memory on the path to Alzheimer's disease. Curr. Opin. Neurobiol. 21, 929-934.

Ghosal, K., Stathopoulos, A., Pimplikar, S.W., 2010. APP intracellular domain impairs adult neurogenesis in transgenic mice by inducing neuroinflammation. PLoS One 5, 1-11.

Gibbs, S.M., 2003. Regulation of neuronal proliferation and differentiation by nitric oxide. Mol. Neurobiol. 27, 107-120.

Gossen, M., Freundlieb, S., Bender, G., Müller, G., Hillen, W., Bujard, H., 1995. Transcriptional activation by tetracyclines in mammalian cells. Science 268, 1766-1769.

Gould, E., Reeves, A.J., Fallah, M., Tanapat, P., Gross, C.G., Fuchs, E., 1999. Hippocampal neurogenesis in adult Old World primates. Proc. Natl. Acad. Sci. U.S.A 96, 5263-5267.

Graham, V., Khudyakov, J., Ellis, P., Pevny, L., 2003. SOX2 functions to maintain neural progenitor identity. Neuron 39, 749-765.

Grasl-Kraupp, B., Ruttkay-Nedecky, B., Koudelka, H., Bukowska, K., Bursch, W., Schulte-Hermann, R., 1995. In situ detection of fragmented DNA (TUNEL assay) fails to discriminate among apoptosis, necrosis, and autolytic cell death: a cautionary note. Hepatology 21, 1465-1468.

Haughey, N.J., Nath, A., Chan, S.L., Borchard, A.C., Rao, M.S., Mattson, M.P., 2002. Disruption of neurogenesis by amyloid beta-peptide, and perturbed neural progenitor cell homeostasis, in models of Alzheimer's disease. J. Neurochem. 83, 1509-1524.

Hong, X.P., Peng, C.X., Wei, W., Tian, Q., Liu, Y.H., Yao, X.Q., Zhang, Y., Cao, F.Y. Wang, Q., Wang, J.Z., 2010. Essential role of tau phosphorylation in adult hippocampal neurogenesis. Hippocampus 20, 1339-1349.

Hu, M., Sun, Y.J., Zhou, Q.G., Chen, L., Hu, Y., Luo, C.X., Wu, J.Y., Xu, J.S., Li, L.X., Zhu, D.Y., 2008. Negative regulation of neurogenesis and spatial memory by NR2B-containing NMDA receptors. J. Neurochem. 106, 1900-1913.

Inokuchi, K., 2011. Adult neurogenesis and modulation of neural circuit function. Curr. Opin. Neurobiol. 21, 360-364.

Ittner, L.M., Ke, Y.D., Delerue, F., Bi, M., Gladbach, A., van Eersel, J., Wölfing, $H$. Chieng, B.C., Christie, M.J., Napier, I.A., Eckert, A., Staufenbiel, M., Hardeman, E. Götz, J., 2010. Dendritic function of tau mediates amyloid-b toxicity in Alzheimer's disease mouse model. Cell 142, 387-397.

Jackson, G.R., Wiedau-Pazos, M., Sang, T.K., Wagle, N., Brown, C.A., Massachi, S., Geschwind, D.H., 2002. Human wild-type tau interacts with wingless pathway components and produces neurofibrillary pathology in Drosophila. Neuron 34 509-519.

Jessberger, S., Romer, B., Babu, H., Kempermann, G., 2005. Seizures induce proliferation and dispersion of doublecortin-positive hippocampal progenitor cells. Exp. Neurol. 196, 342-351.

Kee, N., Teixeira, C.M., Wang, A.H., Frankland, P.W., 2007. Preferential incorporation of adult-generated granule cells into spatial memory networks in the dentate gyrus. Nat. Neurosci. 10, 355-362.

Kempermann, G., Gast, D., Kronenberg, G., Yamaguchi, M., Gage, F.H., 2003. Early determination and long-term persistence of adult-generated new neurons in the hippocampus of mice. Development 130, 391-399.

Kempermann, G., Jessberger, S., Steiner, B., Kronenberg, G., 2004a. Milestones of neuronal development in the adult hippocampus. Trends Neurosci. 27, $447-452$.

Kempermann, G., Wiskott, L., Gage, F.H., 2004b. Functional significance of adult neurogenesis. Curr. Opin. Neurobiol. 14, 186-191. 
Kolb Brown, R., Witt-Lajeunesse, A., Gibb, R., 2001. Neural compensations after lesion of the cerebral cortex. Neural Plast. 8, 1-16.

Kuhn, H.G., Biebl, M., Wilhelm, D., Li, M., Friedlander, R.M., Winkler, J., 2005 Increased generation of granule cells in adult Bcl-2-overexpressing mice: a role for cell death during continued hippocampal neurogenesis. Eur. J. Neurosci. 22, 1907-1915.

Kronenberg, G., Reuter, K., Steiner, B., Brandt, M.D., Jessberger, S., Yamaguchi, M., Kempermann, G., 2003. Subpopulations of proliferating cells of the adult hippocampus respond differently to physiologic neurogenic stimuli. J. Comp. Neurol. 467, 455-463.

Lazarov, O., Mattson, M.P., Peterson, D.A., Pimplikar, S.W., van Praag, H., 2010. When neurogenesis encounters aging and disease. Trends Neurosci 33, 569-579.

Lee, E.Y., Hu, N., Yuan, S.S., Cox, L.A., Bradley, A., Lee, W.H., Herrup, K., 1994. Dual roles of the retinoblastoma protein in cell cycle regulation and neuron differentiation. Genes Dev. 8, 2008-2021.

Lugert, S., Basak, O., Knuckles, P., Haussler, U., Fabel, K., Götz, M., Haas, C.A., Kempermann, G., Taylor, V., Giachino, C., 2010. Quiescent and active hippocampal neural stem cells with distinct morphologies respond selectively to physiological and pathological stimuli and aging. Cell Stem Cell 6, 445-456.

Luo, M.H., Tse, S.W., Memmott, J., Andreadis, A., 2004. Novel isoforms of tau that lack the microtubule-binding domain. J. Neurochem. 90, 340-351.

Mitsuhashi, T., Aoki, Y., Eksioglu, Y.Z., Takahashi, T., Bhide, P.G., Reeves, S.A., Caviness Jr., V.S., 2001. Overexpression of p27Kip1 lengthens the G1 phase in a mouse model that targets inducible gene expression to central nervous system progenitor cells. Proc. Natl. Acad. Sci. U.S.A. 98, 6435-6440.

$\mathrm{Mu}$, Y., Gage, F.H., 2011. Adult hippocampal neurogenesis and its role in Alzheimer's disease. Mol. Neurodegener. 6, 85.

Nácher, J., Varea, E., Miguel Blasco-Ibáñez, J., Gómez-Climent, M.A., CastilloGómez, E., Crespo, C., Martínez-Guijarro, F.J., McEwen, B.S., 2007. N-methyl-daspartate receptor expression during adult neurogenesis in the rat dentate gyrus. Neuroscience $144,855-864$.

Neher, J.J., Neniskyte, U., Zhao, J.W., Bal-Price, A., Tolkovsky, A.M., Brown, G.C., 2011 Inhibition of microglial phagocytosis is sufficient to prevent inflammatory neuronal death. Immunology 186, 4973-4983.

Okano, H.J., Pfaff, D.W., Gibbs, R.B., 1993. RB and Cdc2 expression in brain: correlations with 3H-thymidine incorporation and neurogenesis. J. Neurosci. 13, 2930-2938.

Park, S.Y., Ferreira, A., 2005. The generation of a 17 kDa neurotoxic fragment: an alternative mechanism by which tau mediates beta-amyloid-induced neurodegeneration. J. Neurosci. 25, 5365-5375.

Poppek, D., Keck, S., Ermak, G., Jung, T., Stolzing, A., Ullrich, O., Davies, K.J., Grune, T., 2006. Phosphorylation inhibits turnover of the tau protein by the proteasome: influence of RCAN1 and oxidative stress. Biochem. J. 400, 511-520.

Revest, J.M., Dupret, D., Koehl, M., Funk-Reiter, C., Grosjean, N., Piazza, P.V., Abrous, D.N., 2009. Adult hippocampal neurogenesis is involved in anxietyrelated behaviors. Mol. Psychiatry 14, 959-967.

Rodriguez, J.J., Jones, V.C., Tabuchi, M., Allan, S.M., Knight, E.M., LaFerla, F.M., Oddo, S., Verkhratsky, A., 2008. Impaired adult neurogenesis in the dentate gyrus of a triple transgenic mouse model of Alzheimer's disease. PLoS ONE 3 , e2935.
Rohn, T.T., Rissman, R.A., Davis, M.C., Kim, Y.E., Cotman, C.W., Head, E., 2002. Caspase- 9 activation and caspase cleavage of tau in the Alzheimer's disease brain. Neurobiol. Dis. 11, 341-354.

Saab, B.J., Georgiou, J., Nath, A., Lee, F.J., Wang, M., Michalon, A., Liu, F. Mansuy, I.M., Roder, J.C., 2009. NCS-1 in the dentate gyrus promotes exploration, synaptic plasticity, and rapid acquisition of spatial memory. Neuron 63 643-656.

Sahay, A., Scobie, K.N., Hill, A.S., O’Carroll, C.M., Kheirbek, M.A., Burghardt, N.S. Fenton, A.A., Dranovsky, A., Hen, R., 2011. Increasing adult hippocampal neurogenesis is sufficient to improve pattern separation. Nature 472, 466-470.

Saman, S., Kim, W., Raya, M., Visnick, Y., Miro, S., Saman, S., Jackson, B., McKee, A.C., Alvarez, V.E., Lee, N.C., Hall, G.F., 2012. Exosome-associated tau is secreted in taupathy model and is selectively phosphorylated in cerebrospinal fluid in early Alzheimer disease. J. Biol. Chem. 287, 3842-3849.

Santacruz, K., Lewis, J., Spires, T., Paulson, J., Kotilinek, L., Ingelsson, M., Guimaraes, A., DeTure, M., Ramsden, M., McGowan, E., Forster, C., Yue, M., Orne, J., Janus, C., Mariash, A., Kuskowski, M., Hyman, B., Hutton, M., Ashe, K.H. 2005. Tau suppression in a neurodegenerative mouse model improves memory function. Science 309, 476-481.

Seri, B., Garcia-Verdugo, J.M., McEwen, B.S., Alvarez-Buylla, A., 2001. Astrocytes give rise to new neurons in the adult mammalian hippocampus. J. Neurosci. 21, 7153-7160.

Sennvik, K., Boekhoorn, K., Lasrado, R., Terwel, D., Verhaeghe, S., Korr, H. Schmitz, C., Tomiyama, T., Mori, H., Krugers, H., Joels, M., Ramakers, G.J., Lucassen, P.J., Van Leuven, F., 2007. Tau-4R suppresses proliferation and promotes neuronal differentiation in the hippocampus of tau knockin/knockout mice. FASEB J. 21, 2149-2161.

Sierra, A., Encinas, J.M., Deudero, J.J., Chancey, J.H., Enikolopov, G., OverstreetWadiche, L.S., Tsirka, S.E., Maletic-Savatic, M., 2010. Microglia shape adult hippocampal neurogenesis through apoptosis-coupled phagocytosis. Cell Stem Cell 7, 483-495.

Stone, J.G., Siedlak, S.L., Tabaton, M., Hirano, A. Castellani, R. Santocanale, C. Perry, G., Smith, M.A., Zhu, X., Lee, H.G., 2011. The cell cycle regulator phosphorylated retinoblastoma protein is associated with tau pathology in several tauopathies. J. Neuropathol. Exp. Neurol. 70, 578-587.

Yamaguchi, M., Saito, H., Suzuki, M., Mori, K., 2000. Visualization of neurogenesis in the central nervous system using nestin promoter-GFP transgenic mice. Neuroreport $11,1991-1996$

Yassa, M.A., Stark, C.E., 2011. Pattern separation in the hippocampus. Trends Neurosci. 34, 515-525.

Ünal-Cevik, I., Kilinc, M. Can, A., Gürsoy-Ozdemir, Y., Dalkara, T, 2004. Apoptotic and necrotic death mechanisms are concomitantly activated in the same cell after cerebral ischemia. Stroke 35, 2189-2194.

Zeng, H., Chattarji, S., Barbarosie, M., Rondi-Reig, L., Philpot, B.D., Miyakawa, T., Bear, M.F., Tonegawa, S., 2001. Forebrain-specific calcineurin knockout selectively impairs bidirectional synaptic plasticity and working/episodic-like memory. Cell 107, 617-629.

Zhao, C., Teng, E.M., Summers Jr., R.G., Ming, G.L., Gage, F.H., 2006. Distinct morphological stages of dentate granule neuron maturation in the adult mouse hippocampus. J. Neurosci. 26, 3-11. 